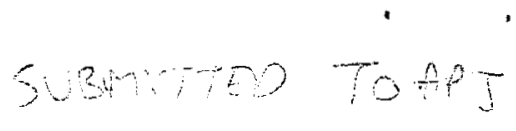

\title{
Analysis of variability in the burst oscillations of the accreting millisecond pulsar XTE J1814-338
}

\author{
Anna L. Watts ${ }^{1}$, Tod E. Strohmayer ${ }^{2}$ and Craig B. Markwardt ${ }^{3}$ \\ X-ray Astrophysics Laboratory, NASA Goddard Space Flight Center, Greenbelt, MD 20771, \\ $U S A$
}

\begin{abstract}
The accreting millisecond pulsar XTE J1814-338 exhibits oscillations at the known spin frequency during Type I X-ray bursts. The properties of the burst oscillations reflect the nature of the thermal asymmetry on the stellar surface. We present an analysis of the variability of the burst oscillations of this source, focusing on three characteristics: fractional amplitude, harmonic content and frequency. Fractional amplitude and harmonic content constrain the size, shape and position of the emitting region, whilst variations in frequency indicate motion of the emitting region on the neutron star surface. We examine both long-term variability over the course of the outburst, and short-term variability during the bursts. For most of the bursts, fractional amplitude is consistent with that of the accretion pulsations, implying a low degree of fuel spread. There is however a population of bursts whose fractional amplitudes are substantially lower, implying a higher degree of fuel spread, possibly forced by the explosive burning front of a precursor burst. For the first harmonic, substantial differences between the burst and accretion pulsations suggest that hotspot geometry is not the only mechanism giving rise to harmonic content in the latter. Fractional amplitude variability during the bursts is low; we can only rule out the hypothesis that the fractional amplitude remains constant at the $1 \sigma$ level for bursts that do not exhibit photospheric radius expansion (PRE). There are no significant variations in frequency in any of the bursts except for the one burst that exhibits PRE. This burst exhibits a highly significant but small $(\approx 0.1 \mathrm{~Hz})$ drop in frequency in the burst rise. The timescale of the frequency shift is slower than simple burning layer expansion models predict, suggesting that other mechanisms may be at work.
\end{abstract}

\footnotetext{
1National Research Council Resident Research Associate; anna@milkyway.gsfc.nasa.gov

${ }^{2}$ stroh@clarence.gsfc.nasa.gov

${ }^{3}$ Department of Astronomy, University of Maryland, College Park, MD 20772; craigm@milkyway.gsfc.nasa.gov
} 
Subject headings: binaries: general, stars: individual (XTE J1814-338), stars: neutron, stars: rotation, X-rays: bursts, X-rays: stars

\section{Introduction}

There are now six known accreting millisecond pulsars: SAX J1808.4-3658 (Wijnands \& van der Klis 1998; Chakrabarty \& Morgan 1998); XTE J1751-305 (Markwardt et al. 2002); XTE J0929-314 (Galloway et al. 2002); XTE J1807-294 (Markwardt, Smith \& Swank 2003); XTE J1814-338 (Markwardt \& Swank 2003); and IGR J00291+5934 (Galloway et al. 2005). Of these six only two, SAX J1808.4-3658 (hereafter J1808) and XTE J1814-338 (hereafter J1814), have shown X-ray bursts. The bursts of both systems show oscillations at or very close to the known spin frequency of the neutron star (Chakrabarty et al. 2003; Strohmayer et al. 2003). This suggests that rotation is modulating an asymmetry on the burning surface that is near-stationary in the co-rotating frame. The nature of the asymmetry, however, remains unresolved.

In the case of J1808 and J1814 we know that fuel deposition is inhomogeneous, since otherwise we would not observe the systems as pulsars. One thing that is not clear, however, is whether the material builds up at the deposition point or whether it spreads out over the surface of the star, thereby lessening asymmetries in fuel coverage (Inogamov \& Sunyaev 1999). In either case, however, coverage is unlikely to be completely even. This means that ignition is likely to occur at a particular point rather than occurring simultaneously across the entire surface. As the burning front spreads to engulf the available fuel (giving an overall rise in luminosity), changes in the emitting region should be reflected in the properties of the burst oscillations.

There are several mechanisms that may give rise to a thermal asymmetry. If there are significant inhomogeneities in fuel coverage, caused for example by magnetic channelling, areas with more fuel should get hotter. These thermal asymmetries may then persist as the surface of the star cools in the burst tail. If fuel coverage is even, the initial hotspot that develops at the ignition point is expected to grow rapidly to engulf the whole star during the burst rise. If this is the case, what then causes the asymmetry in the burst tail? Possibilities include the development of vortices driven by the Coriolis force (Spitkovsky, Levin \& Ushomirsky 2001), or a brightness pattern caused by oscillations in the surface layers (McDermott \& Taam 1987; Lee 2004; Heyl 2004; Piro \& Bildsten 2005). Mechanisms that do not rely on fuel channelling are of particular importance for the non-pulsing bursters, systems for which there is no evidence for asymmetric fuel deposition. By attempting to distinguish the candidate mechanisms we hope to probe not only the nuclear burning process and the 
surface layer composition, but also the role of the magnetic field in controlling the accretion flow and the motion of the surface layers.

The transient system J1814 was first observed in outburst on June 3rd 2003 by the Rossi $X$-ray Timing Explorer (RXTE) Galactic bulge monitoring campaign. A longer observation on June 5th confirmed that the source was a pulsar (Markwardt \& Swank 2003). The pulsar has a spin frequency of $314.36 \mathrm{~Hz}$, resides in a binary with an orbital period of $4.275 \mathrm{hr}$, and has a minimum companion mass of $\approx 0.15 M_{\odot}$ (Markwardt et al 2005, in preparation). J1814 is the widest and most massive binary of the known accreting millisecond pulsars: in this regard it is the most similar to the non-pulsing low mass X-ray binaries (LMXBs). The system remained in outburst until mid July 2003. Over this period repeated observations were made with the RXTE Proportional Counter Array (PCA), during which 28 Type I $\mathrm{X}$-ray bursts were recorded. A preliminary analysis of the bursts was given in Strohmayer et al. (2003).

In this paper we present an analysis of the variability of the burst oscillations of J1814. We focus on three particular characteristics: fractional amplitude, harmonic content, and Irequency. Fractional amplitude and harmonic content constrain the size, shape and position of the emitting region, whilst any changes in frequency over and above those expected for orbital corrections indicate motion of the emitting region on the stellar surface. We examine both short-term variations during bursts, and long-term variations in burst properties over the course of the outburst. The short-term variability reveals how the size, shape and position of the emitting regions evolve during the thermonuclear burst. The long-term variations reflect the influence of the accretion and burning history. This history is likely to affect both the magnetic field (which may be suppressed as the outburst proceeds, affecting fuel deposition; Cumming, Zweibel \& Bildsten (2001)) and the composition of the surface (as successive thermonuclear bursts process the accreted material into heavier elements; Taam (1980); Woosley et al. (2004)).

Section 2 gives an overview of our method of analysis. In Section 3 we review the general characteristics of the outburst. Section 4 details our analysis of the variability of the burst oscillations. Section 5 discusses the results and relates them to current theories of burst oscillation mechanisms. We conclude with brief comments on issues requiring further study. 


\section{Method of analysis}

\subsection{Computation of fractional amplitude and frequency}

Our analysis of $J 1814$ was conducted using $125 \mu$ s time resolution PCA event mode data. We first barycentered the data using the JPL DE405 ephemeris and the source position determined from PCA scans (Markwardt \& Swank 2003; Krauss et al. 2005). Fractional amplitudes, frequencies and harmonic content were then analysed using two complementary techniques: the $Z_{n}^{2}$ statistic (Buccheri et al. 1983; Strohmayer \& Markwardt 2002); and pulse profile fitting.

The $Z_{n}^{2}$ statistic is very similar to the standard power spectrum computed from a Fourier transform, but is used when one is dealing with event data instead of binned data. It is defined as:

$$
Z_{n}^{2}=\frac{2}{N} \sum_{k=1}^{n}\left[\left(\sum_{j=1}^{N} \cos k \phi_{j}\right)^{2}+\left(\sum_{j=1}^{N} \sin k \phi_{j}\right)^{2}\right]
$$

where $n$ is the number of harmonics, $N$ is the total number of photons, and $j$ is an index applied to each photon. The phase $\phi_{j}$ calculated for each photon is

$$
\phi_{j}=2 \pi \int_{t_{0}}^{t_{j}} \nu(t) d t
$$

where $\nu(t)$ is the frequency model, and $t_{j}$ the arrival time of the photon relative to some reference time. The rms fractional amplitude $r$ for the trial frequency is then given by

$$
r=\left(\frac{\bar{Z}_{n}^{2}}{N_{s}}\right)^{1 / 2}
$$

where $N_{s}$ is the number of source photons, and $\bar{Z}_{n}^{2}$ is the $Z_{n}^{2}$ statistic for the source alone. The statistic that we compute from the data, using equation (1), includes all photons: $N_{s}$ from the source; and $N_{b}$ from the background. If we assume that the background photons are not periodic for the range of trial frequencies investigated, the background makes no contribution to the term in the bracket in equation (1). In this case

Equation (3) becomes

$$
\bar{Z}_{n}^{2}=\frac{N}{N-N_{b}} Z_{n}^{2}
$$




$$
r=\left(\frac{Z_{n}^{2}}{N}\right)^{1 / 2}\left(\frac{N}{N-N_{b}}\right)
$$

The number of background photons, $N_{b}$, can be estimated using the standard FTOOLS routine pcabackest and the PCA background models.

We have now corrected for the background, but we have yet to account for the effects of noise. If the true signal power (as calculated using the $Z_{n}^{2}$ statistic) is $Z_{s}$, then the measured values $Z_{m}$ will be distributed according to

$$
p_{n}\left(Z_{m}: Z_{s}\right)=\frac{1}{2} \exp \left[-\frac{\left(Z_{m}+Z_{s}\right)}{2}\right]\left(\frac{Z_{m}}{Z_{s}}\right)^{(n-1) / 2} I_{n-1}\left(\sqrt{Z_{m} Z_{s}}\right)
$$

This distribution can be derived following the procedure outlined by Groth (1975) for binned data, modified to use the normalisation of noise power used by Leahy et al. (1983), as discussed by Vaughan et al. (1994) and Muno, Özel \& Chakrabarty (2002). Example distributions are shown in Figure 1; note that the distribution differs significantly from a normal distribution, particularly for weak signals. We have confirmed the accuracy of this distribution for a trial frequency model using Monte Carlo simulations to generate events files where the model light curve includes a periodic signal. The expectation value and variance of $Z_{m}$ are given by

$$
\begin{gathered}
\left\langle Z_{m}\right\rangle=Z_{s}+2 n \\
\left\langle\left(Z_{m}-\left\langle Z_{m}\right\rangle\right)^{2}\right\rangle=4\left(Z_{s}+n\right)
\end{gathered}
$$

The probability of obtaining a measured $Z_{n}^{2}$ that lies between 0 and $Z_{m}$, given $Z_{s}$, is given by the associated cumulative distribution function:

$$
f_{n}\left(Z_{m}: Z_{s}\right)=1-\exp \left[-\frac{\left(Z_{m}+Z_{s}\right)}{2}\right]\left[\sum_{k=0}^{\infty} \sum_{l=0}^{k+n-1} \frac{\left(Z_{s}\right)^{k}\left(Z_{m}\right)^{l}}{l ! k ! 2^{k+l}}\right]
$$

The probability of the true signal power lying between 0 and $Z_{s}$ given a measured power $Z_{m}$ is then given by

$$
f_{n}\left(Z_{s}: Z_{m}\right)=1-f_{n}\left(Z_{m}: Z_{s}\right)
$$

It is $Z_{s}$ that we want to use in equation (5); we use equation (10) to infer this quantity from $Z_{m}$. In this paper we take the best estimate of $Z_{s}$ to be the value for which $f_{n}\left(Z_{s}: Z_{m}\right)=0.5$. 
This is a matter of choice, something that must be borne in mind when interpreting the results. A reasonable alternative, for example, would be to pick the $Z_{s}$ for which $p\left(Z_{m}: Z_{s}\right)$ is a maximum. In computing the error bars that appear in plots of fractional amplitude in this paper the errors on $Z_{s}$ are taken to be the points $f_{n}\left(Z_{s}: Z_{m}\right)=0.159$ and $f_{n}\left(Z_{s}: Z_{m}\right)=$ 0.841 (equivalent to $\pm 1 \sigma$ for a normal distribution).

One other issue that arises during the bursts is that there are two contributions to the pulsations: asymmetries due to accretion (which give rise to the pulsations observed between bursts) and asymmetries due to the thermonuclear process. If we make the assumption that accretion continues during the burst, the measured fractional amplitude will contain contributions from both processes:

$$
r=\frac{r_{\mathrm{bur}} N_{\mathrm{bur}}+r_{\mathrm{acc}} N_{\mathrm{acc}}}{N_{s}}
$$

$N_{\text {bur }}$ and $N_{\text {acc }}$ are the number of source photons arising from the burst and accretion processes respectively, with $r_{\text {bur }}$ and $r_{\text {acc }}$ being the fractional amplitudes of the two different processes. The total number of source photons, $N_{s}=N_{\text {bur }}+N_{\text {acc }}$. If $N_{\text {bur }} \gg N_{\text {acc }}$ then $r \approx r_{\text {bur }}$, but we will not always be in this regime, so we will need to estimate $N_{\text {acc }}$ and $r_{\text {acc }}$ in order to isolate $r_{\text {bur. }}$.

An alternative method of analysis is pulse profile fitting. As with the $Z_{n}^{2}$ statistic, we first pick a trial frequency model and assign a phase to each event. The events are then allocated to phase bins; by recording the number of events in each bin we can generate a pulse profile. To the resulting binned data we fit a function of the form:

$$
F(\phi)=A+\sum_{k=1}^{n} a_{n} \sin \left[k\left(\phi+b_{n}\right)\right]
$$

where $n$ is the number of harmonics fitted. The parameters $A, a_{n}$ and $b_{n}$ are adjusted and the best fit found by minimising $\chi^{2}=\sum_{i=1}^{N_{\phi}}\left[g_{i}-F\left(\phi_{i}\right)\right]^{2} / g_{i}$, where $\phi_{i}$ is the phase in the $i$ th bin, $N_{\phi}$ is the number of phase bins, and $g_{i}$ is the number of events in that phase bin. The rms fractional amplitude for that trial frequency model is given by $a_{n} / \sqrt{2}$.

All of the results presented in this paper were derived using the $Z_{n}^{2}$ method. However, we ran an extensive series of tests using the profile fitting method, and verified that the results agreed to within $1 \%$ of those acquired using the $Z_{n}^{2}$ method. 


\section{General characteristics of the outburst}

In order to understand the variability of the X-ray bursts it is important to understand the context in which they take place. In this section we review the general characteristics of the 2003 outburst insofar as they influence the bursts.

\subsection{Accretion rate}

A key factor affecting burst frequency and composition is the local accretion rate (see Strohmayer \& Bildsten (2005) and references therein). An indicator of variations in the accretion rate is the non-burst flux, shown in the upper panel of Figure 2. Galloway, Cumming \& Chakrabarty (2004) have used spectral fitting to estimate the bolometric flux for the 2003 outburst of J1814. Using the distance estimate of $\approx 8 \mathrm{kpc}$ (Strohmayer et al. 2003), they infer that the peak accretion during the outburst corresponds to $\approx 4 \% \dot{M}_{\text {Edd }}$. A corresponding lower bound for the local accretion rate, $\dot{m}$, can be calculated by assuming that material is deposited evenly across the stellar surface: $\dot{m}>3 \times 10^{3} \mathrm{~g} \mathrm{~cm}^{-2} \mathrm{~s}^{-1}$. Accretion at this rate suggests that the bursts of $\mathrm{J} 1814$ should be mixed $\mathrm{H} / \mathrm{He}$ bursts triggered by unstable helium ignition (Fujimoto, Hanawa \& Miyaji 1981; Fushiki \& Lamb 1987; Cumming \& Bildsten 2000). For bursts taking place in the lower accretion rate regime after MJD 52830, one might expect both a drop in burst rate and more stable hydrogen burning, giving rise a higher helium fraction in the bursts.

We note that position in the color-color diagram is cited as a more reliable indicator of variations in accretion rate than X-ray flux (van der Klis 1995). Analysis of the 2003 outburst of J1814 shows it to be in the extreme island state, with the source moving to the left in a plot of hard vs soft color as the countrate declines after MJD 52830 (van Straaten, van der Klis \& Wijnands 2005). For brighter sources this would ordinarily signify an increase in accretion rate, but for such a weak transient source movement within the island state is not well studied, so we will assume that the drop in flux represents a genuine drop in accretion rate.

Figure 3 shows the percentage daily coverage achieved by the RXTE PCA during the outburst, with the burst detection rate shown for comparison. Given the coverage, the burst detection rate suggests a burst rate of $6-8$ per day in the peak of the outburst, with a lower rate of $4-6$ per day when the accretion rate is lower at the start and tail of the outburst (in accordance with the burst recurrence times suggested by Galloway, Cumming \& Chakrabarty (2004)). A decrease in burst rate as accretion rate drops is in accordance with theoretical expectations, assuming that the fuel deposition footprint does not vary substantially over 
the course of the outburst.

All of the bursts have rise times in the range 1 to $8 \mathrm{~s}$, with total burst duration 100 to $200 \mathrm{~s}$. This is consistent with all of the bursts being mixed $\mathrm{H} / \mathrm{He}$ bursts. However, the burst luminosity varies strongly from burst to burst (Figure 4). The bursts with the shortest rise times tend to be brighter (Strohmayer et al. (2003); Figure 5), suggesting that there may be a higher proportion of helium in the burning mix. A high proportion of helium in the final burst is not unexpected (due to the lower accretion rate), but it is notable that the brightness seems to vary even during a regime of relatively constant accretion rate. The burst recurrence times are also variable (Galloway, Cumming \& Chakrabarty 2004). Such variations could occur naturally if different areas on the star are igniting. Alternatively we could be seeing the effects of thermal or compositional inertia, where the burning history affects subsequent bursts (Taam 1980; Woosley et al. 2004). Galloway, Cumming \& Chakrabarty (2004) also noted that burst recurrence times are shorter than would be expected if accretion were uniform across the stellar surface. This suggests that local accretion rate is higher than the minimum value calculated elsewhere in this section, most probably due to channelling of the accretion flow.

\subsection{Fuel deposition pattern}

X-ray emission from an accreting neutron star contains contributions from the accretion disk, the stellar surface, and the boundary layer immediately above the surface. Asymmetries in the resulting luminosity pattern give rise to the non-burst pulsations. To understand what the fractional amplitude of these pulsations tells us about fuel deposition, we need to understand the contribution of the different components to the X-ray emission.

In the 2003 outburst J1814 was in the island state, with relatively low accretion rate. Done \& Gierliński (2003) argue that in this case the inner edge of the disk is likely to be far from the star, in which case the bulk of the emission from the accretion disk is likely to be outside the PCA bandpass. If the PCA emission is dominated by the contribution from the stellar surface then the fractional amplitude and harmonic content of the non-burst pulsations are a direct probe of the pattern of fuel deposition on the stellar surface. If on the other hand the disk contribution cannot be neglected, the fractional amplitude of the surface component will be higher than that measured (assuming that the disk emission is symmetric).

Asymmetries are thought to arise because some of the matter from the accretion disk is channelled out of the disk and along magnetic field lines towards the magnetic poles. 
The degree of channelling, which we define as the difference between the minimum and maximum local accretion rates, is a matter of debate. Given the relatively weak magnetic fields of the accreting millisecond pulsars, at least some of the matter is likely to penetrate the field lines and accrete in a spherically symmetric fashion (see for example Scharlemann (1978); Ghosh \& Lamb (1979); Spruit \& Taam (1990); Miller, Lamb \& Psaltis (1998)). X-ray generation will involve thermal emission from the neutron star surface (as accreted matter is processed), reflection of photons from the surface, and possible emission from shocked plasma in the magnetic accretion funnel immediately above the stellar surface (Basko \& Sunyaev 1976; Lyubarskii \& Sunyaev 1982; Gierliński, Done \& Barret 2002; Done \& Gierliński 2003). Spectral analysis and modelling have been applied to J1808 in an effort to tease out the various contributions (Gilfanov et al. 1998; Gierliński, Done \& Barret 2002; Poutanen \& Gierlinski 2003), but a detailed study of this type has yet to be done for J1814.

Regardless of the precise emission mechanism, however, the fractional amplitude of the non-burst pulsations will reflect the amount of material deposited in the region of the magnetic polar caps as compared to the rest of the stellar surface. The effect of different deposition geometries is illustrated in Figure 6 . If the fractional amplitude is very high we must have a bright hotspot on an otherwise dim surface (strong channelling), and the geometry must be such that the hotspot moves out of the field of view as the pulsar rotates (upper panel, Figure 6). If fractional amplitude is lower we have two possibilities. The first is that channelling is weak, leading to reasonably bright emission from much of the stellar surface. In this case there will be no major changes in brightness as the pulsar rotates (center panel, Figure 6). The second is that channelling is strong and most of the star is dim, but that spot size and observer inclination are such that the projected area of the hotspot is never zero (lower panel, Figure 6).

The strength of any harmonics may allow us to distinguish these possibilities. Harmonic content may arise in several ways: firstly if the geometry is such that the second antipodal magnetic cap is also visible; secondly due to the Doppler shifts, which are more pronounced if the spot is close to the rotational equator; and thirdly due to the effects of beaming or a non-spherical accretion footprint.

The lower two panels of Figure 2 show the evolution of fractional amplitudes of the fundamental and first harmonic of the pulsar frequency during the non-burst emission. These values were calculated according to the prescription outlined in Section 2 (see Cui, Morgan \& Titarchuk (1998) for similar plots for J1808). Daily values of $Z_{m}$ were calculated using all good events from a given day. The frequency model used was the best fit binary orbital ephemeris (Markwardt et al 2005, in preparation). The fractional amplitude of the fundamental, at $\approx 10 \%$, is relatively low, suggesting one of the two possible low amplitude 
geometries.

We do not attempt a comprehensive study of the non-burst pulsations in this paper. We include the results presented for two reasons. Firstly, we make the assumption that the accretion process that gives rise to the non-burst pulsations continues even during the bursts. We need to take into account this contribution to the overall fractional amplitude when calculating the fractional amplitudes due to the thermonuclear burst process. Secondly we wish to compare the fractional amplitudes and harmonic content of the bursts with those of the non-burst pulsations. If fractional amplitude is substantially lower during the bursts, for example, this would suggest that fuel is spreading over the surface of the star after accretion.

\section{Burst oscillation variability}

\subsection{Variability over the course of the outburst}

In this section we consider variation in the average properties of each burst over the course of the outburst. We define the burst start and end times as being the times between which the count rate consistently exceeds the average persistent rate. All photons arriving between those times are considered to be associated with the burst, and the analysis methods are applied to the set of photons as a whole. Table 1 summarises average burst properties for the set of bursts.

Strohmayer et al. (2003) showed that the burst oscillation frequency was consistent with the pulsar frequency, and that frequency shifts during the bursts were very small. In calculating burst average fractional amplitudes we therefore neglect any additional frequency shifts and take as our frequency model the best fit orbital ephemeris. We will discuss frequency variability during the bursts in more detail in Section 4.2.

To isolate the fractional amplitude associated with the burst process, one needs to know the accretion luminosity and the accretion fractional amplitude (equation 11). We can estimate these quantities by analysing segments of data immediately preceding or following the bursts. One problem with this approach is that one has to assume that the accretion flux and fractional amplitude remain steady over timescales of $\sim 100 \mathrm{~s}$. Analysis of the non-burst pulsations indicates that statistical fluctuations alone can lead to these quantities varying routinely by $10-20 \%$. For the fainter bursts, where $N_{\text {acc }} \approx N_{\text {bur }}$, this could introduce errors of this magnitude into our calculation of burst fractional amplitude.

To minimise the effect of variations (statistical or intrinsic) in the accretion related 
pulsations we calculate fractional amplitudes using only events that occur when the flux is at least twice the pre-burst average. By setting such a threshold we ensure that $N_{\text {acc }} / N_{\text {bur }}<$ 0.25 for all of the bursts. This makes $r$ a better approximation to $r_{\text {bur }}$, and reduces the effect of variations in $N_{\text {acc }}$ and $r_{\text {acc }}$. Accretion corrections will still be important, however, if the estimated $r_{\text {acc }}$ differs substantially from the measured $r$.

Figure 7 shows the burst fractional amplitude at the fundamental frequency of the oscillations over the course of the outburst. Corresponding results for the first harmonic are shown in Figure 8. Accretion corrections have little effect on the fundamental fractional amplitudes, but they have a noticeable effect on the first harmonic fractional amplitudes.

Let us first consider whether there is genuine variation in the burst fractional amplitude over the course of the outburst. In other words, are the data consistent with a burst fractional amplitude that remains constant over the course of the outburst? We start by defining a simple measure of amplitude variability:

$$
\bar{\chi}^{2}=\sum_{i}^{N_{\mathrm{tu} i}}\left(r_{i}-r_{\text {model }}\right)^{2}
$$

$N_{\text {bur }}$ is the number of bursts, $r_{i}$ is the fractional amplitude calculated for a particular burst, and $r_{\text {model }}$ is the trial amplitude model, in this case a constant. We compute this quantity for the data for a range of trial fracional amplitudes. In contrast with the more familiar $\chi^{2}$ statistic we do not weight by the errors because they are non-Gaussian and non-symmetric. Instead we will use Monte Carlo simulations to determine the distribution of $\bar{\chi}^{2}$ under the constant fractional amplitude hypothesis and estimate the significance of the result.

We therefore need to simulate $N_{\text {sim }}$ sets of $N_{\text {bur }}$ fractional amplitude measurements. For each burst $Z_{s}$, and hence the distribution of fractional amplitudes, is different (because $N_{s}$ and $N_{b}$ are different), the distribution being wider for lower $Z_{s}$. Rather than generating artificial events files for each burst, we can make use of the fact that we know the cumulative distribution function $f\left(Z_{m}: Z_{s}\right)$. If we are running $N_{\text {sim }}$ simulations we start by generating $N_{\text {bur }} \times N_{\text {sim }}$ random numbers drawn from a uniform distribution between 0 and 1 . These are our values of $f$. For each burst we take $N_{\text {sim }}$ of these $f$ values, compute the appropriate $Z_{s}$ for the trial fractional amplitude, and solve equation (9) numerically to generate $N_{\text {sim }}$ values of $Z_{m}$. We then proceed exactly as we did for the data and compute fractional amplitudes. We have now simulated the distribution of $r$ for each burst under the constant fractional amplitude hypothesis.

We then group the simulated data into sets of $N_{\text {bur }}$, each set containing one point from each different burst, and compute $N_{\text {sim }}$ values of $\bar{\chi}^{2}$. We repeat this exercise for the full 
range of trial fractional amplitudes. By computing the number of simulations in which the simulated $\bar{\chi}^{2}$ exceeds the measured value we can determine the significance of the measured variability.

We start with the fractional amplitude at the fundamental frequency of the oscillations. If we consider the full set of 28 bursts we find that the simulated $\bar{\chi}^{2}$ does not exceed the measured value once even after simulating $10^{4}$ sets of bursts. This result holds true for both accretion corrected and non-corrected values, and constitutes a strong rejection of the constant fractional amplitude hypothesis.

What about relaxing the hypothesis? The most obvious outlier is the data point from the last burst. This burst takes place when the accretion rate has dropped substantially, and it is plausible that the fractional amplitude may indeed change for this burst. Strohmayer et al. (2003) also found evidence that this burst exhibits photospheric radius expansion, which would have the effect of suppressing the fractional amplitude. We therefore relax our hypothesis and ask how likely it is that all of the bursts except for the last one have a constant fractional amplitude. This reduces the measured $\bar{\chi}^{2}$ substantially, but the simulated $\bar{\chi}^{2}$ still never exceeds the measured value once even after $10^{4}$ simulations. The constant fractional amplitude hypothesis is still rejected. There is genuine variation in burst fractional amplitude.

Note that in this analysis we have taken no account of the temporal order of the measurements, despite the fact that there seem to be more values below the best fit fractional amplitude at the start of the outburst. We are reluctant to make any pronouncement about whether or not this is statistically significant. Our caution stems from the fact that RXTE is likely to have missed many bursts, particularly in the second part of the outburst, where daily coverage dropped dramatically (see Figure 3). To make any progress in a temporal analysis one would need even coverage across the outburst.

For the first harmonic the simulated $\bar{\chi}^{2}$ exceeds the measured value for the full set of bursts in 31 simulations out of 1000 (for the non-corrected values), the best fit amplitude being $1.95 \%$. This constitutes a rejection of the hypothesis at a level just short of $2 \sigma$. If we exclude the last burst from the set, the simulated $\bar{\chi}^{2}$ exceeds the measured value in 390 simulations out of 1000 simulations, with best fit amplitude $2 \%$, and the hypothesis cannot be ruled out at all. The strength of the rejection in both cases falls if we include the accretion correction: the best fit amplitude excluding the last burst is shown in Figure 9 .

We make one additional comment. In the later part of the outbursts there are fewer bursts above the best fit line than in the earlier stages. Bhattacharyya et al. (2005) noted this apparent reduction in harmonic content in their paper. One should however be very 
cautious here because of the fall in coverage in the later phases of the outburst. Excluding the last burst (which takes place in a dramatically different regime) the data are well fit by a constant fractional amplitude, and confirmation that there is a definite drop in harmonic content requires more observations in the tail of the outburst.

We also want to test whether the burst fractional amplitudes are the same as the daily average accretion fractional amplitude at the time of each burst. In other words, we want to test the hypothesis that the fractional amplitude remains constant for a given day irrespective of the emission mechanism. This would indicate a low degree of fuel spread from the deposition point.

In testing this hypothesis we must take into account the fact that there is uncertainty in our measurement of daily average fractional amplitude, as well as in our measurements of burst fractional amplitudes. We proceed as follows. For a given day we have one or two values of fractional amplitude calculated during bursts, and one value of fractional amplitude formed by folding together non-burst data. We start by identifying, for each day, the fractional amplitude $r_{m}$ that maximises the likelihood of obtaining both the burst and non-burst measurements made during that day. The likelihood $L$ is defined as:

$$
L=\prod_{i} p_{i}\left(Z_{m}: Z_{s}\left(r, N, N_{b}\right)\right)
$$

where the product contains the two or three data points that we have for each day (Wall $\&$ Jenkins 2003).

Having identified the most probable fractional amplitude $r_{m}$ for each day, we proceed as we did for the constant fractional amplitude case, computing a variability measure for the data and comparing it to simulations. This time however we must include the non-burst data points in our test: both the burst and non-burst measurements are equally valid tests of the hypothesis that daily fractional amplitude is constant irrespective of mechanism.

We compute two different measures of variability. The first is just an amended version of the $\bar{\chi}^{2}$ defined in equation (13):

$$
\bar{\chi}^{2}=\sum_{i}^{N_{\text {bur }}+N_{\text {day }}}\left(r_{i}-r_{\text {model }}\right)^{2}
$$

where $N_{\text {day }}$ is the number of days on which bursts were observed, for which we have computed non-burst fractional amplitude measurements. In this case $r_{\text {model }}$ is the value of $r_{m}$ appropriate to each data point. This measure checks overall scatter of burst and non-burst points. It does not take into account the sign of the difference. As such, it will not pick out 
unusual behavior such as the burst amplitudes always lying below the best fit value, with the accretion values always lying above. For this reason we also compute a second measure for the burst values alone:

$$
\bar{\chi}=\sum_{i}^{N_{\text {bur }}}\left(r_{i}-r_{\text {model }}\right)
$$

As before, we run Monte Carlo simulations to determine the distribution of both of our measures under the assumption that the hypothesis is correct.

Figure 10 shows the degree of overlap between the burst and non-burst fractional amplitudes for the fundamental. Performing the analysis on the full set of bursts, the hypothesis that the bursts have the same fractional amplitude as the persistent emission is rejected at a level greater than one part in $10^{4}$. The figure illustrates why this is the case. For most of the bursts, there is in fact rather good agreement between the burst and non-burst fractional amplitude. There are however 6 bursts with fractional amplitudes that are substantially lower than the persistent fractional amplitude. These are bursts 1, 6, 9, to a lesser extent bursts 26 and 27 , and of course burst 28 .

The fractional amplitude in burst 28 is thought to be suppressed because of photospheric radius expansion. If we assume that the true fractional amplitude at the surface is simply obscured by the photosphere, we can re-compute fractional amplitudes neglecting events from the peak of the burst. In this case we obtain a burst average fractional amplitude that is higher (at approximately $6 \%$ ), but which is still substantially lower than the persistent value. A simple explanation for these bursts is that the fuel has spread further from the deposition point. For Burst 28, where recurrence time has increased, this is perhaps reasonable. For the other bursts, it is hard to understand why only a subset of the bursts are affected if the only factor affecting spread is the time elapsed since the last burst. One possibility is that the explosive burning front of the previous burst has forced fuel out across the stellar surface. The nature of the precursor burst would then be an important determining factor, but this is hard to verify given the gaps in the current data.

Figure 11 shows the degree of overlap between burst and non-burst fractional amplitudes for the first harmonic. Our analysis shows that once again the hypothesis that the burst have the same fractional amplitude as the persistent emission can be rejected at a level greater than one part in $10^{4}$. The most stringent rejection is provided by the $\bar{\chi}$ test (equation 16). It is clear from the figure that the fractional amplitude of the first harmonic in the bursts is, for all but 3 of the bursts, lower than the non-burst amplitude. Moreover, the trend of reducing first harmonic content seen in the non-burst pulsations is not reflected in the burst oscillations. This suggests that some mechanism other than hotspot geometry contributes 
to the harmonic content of the non-burst pulsations.

We have also analysed the data to determine whether there is any interesting relationship between fractional amplitude and burst flux. Figures 12 and 13 show that fractional amplitudes of both the fundamental and first harmonic seem to be relatively independent of burst flux. Two features merit comment. The first is the very low fractional amplitude for the burst with the highest peak flux, Burst 28. As discussed by Strohmayer et al. (2003), this burst shows evidence for photospheric radius expansion, which would have the effect of suppressing fractional amplitude. The second point is more speculative. The set of fainter bursts includes three with rather low fundamental fractional amplitudes compared to the rest of the population. This could be due to statistical effects, since the uncertainties on fractional amplitudes for the fainter bursts are higher. However, we may be seeing a sample of a population that has a genuinely lower fractional amplitude: it would be interesting to look for more bursts of this type if J1814 goes into outburst again.

\subsection{Variability during bursts}

Strohmayer et al. (2003) noted that the bursts of J1814 seemed to show variations in rms fractional amplitude of $\approx 3-5 \%$, on timescales of 7 to $15 \mathrm{~s}$. In this section we present an in-depth analysis of this variability. We focus only on the benavior of the fundamentai; the first harmonic is sufficiently weak that we cannot hope to make reasonable detections on shorter timescales than those analysed in section 4.1 .

The upper panels of Figure 14 show the light curves and fractional amplitude variations for a sample of the bursts. The burst average fractional amplitude is also shown for comparison. Fractional amplitudes are calculated only when the flux is at least twice the pre-burst level (to reduce the contribution of the accretion component to any variability). We use non-overlapping bins of 5000 photons. The durations of these bins are sufficiently short that we assume frequency to be constant during each bin, and select the frequency $\nu_{m}$ for which $Z_{m}$ is maximised. The effect of this assumption on the distribution of $Z_{m}$ values is discussed below.

The fractional amplitude during the bursts does seem to vary around the burst average value, with the difference between maximum and minimum fractional amplitudes measured during a burst lying in the range 2.4 to $9.9 \%$. As in Section 4.1, we want to know whether the observed variability is consistent with that which we might expect due to statistical effects alone. The null hypothesis is that fractional amplitude remains constant over the course of a burst. To quantify the variability we evaluate the following quantity for each burst: 


$$
\bar{\chi}^{2}=\sum_{i}^{N_{\text {bins }}}\left(r_{i}-r_{\text {model }}\right)^{2}
$$

$N_{\text {bins }}$ is the number of non-overlapping 5000 photon bins for a given burst and $r_{i}$ is the fractional amplitude calculated for that bin. In this case $r_{\text {model }}$ is a constant that is varied until $\bar{\chi}^{2}$ is minimised.

To simulate the distribution of $\bar{\chi}^{2}$ if the hypothesis is true, in order to estimate the significance of the measured value, we proceed as follows. For each burst we fit the light curve, and generate simulated events files, imposing a constant fractional amplitude oscillation on the light curve. The fractional amplitude assumed for each burst is the burst average fractional amplitude calculated in the previous section and listed in Table 1 . We then analyse the simulated events files in exactly the same way as we analyse the real data, taking 5000 photon segments and calculating a $Z_{m}$ for each segment. We then compute the value of $Z_{s}$ for which $f\left(Z_{m}: Z_{s}\right)=0.5$ and use this to calculate a fractional amplitude for the segment. We run 1000 simulations for each burst and compute the quantity $\bar{\chi}^{2}$ for each simulations. For each burst we can then determine the probability $V_{r}$ that the simulated $\bar{\chi}^{2}$ exceeds the measured value. This gives us a simple measure of the variability of each burst. The results are given in Table 1 and Figure 15. Note that for Burst 28 we ran additional (104) simulations to confirm the high variability of this burst.

A legitimate question is why we used simulated light curves to generate the distribution of fractional amplitudes, rather than adopting the approach that we took in Section 4.1 . We do this because we need to take into consideration the effect of allowing a degree of freedom in frequency selection. Choosing the frequency that maximises $Z_{m}$ has the effect of skewing $p\left(Z_{m}: Z_{s}\right)$ away from the theoretical distribution. The effect is not large, but the peak of the distribution moves to a value of $Z_{m}$ that is a few units higher than the theoretical prediction. That this is to be expected can be seen by considering the distribution in the absence of any signal. Running Monte Carlo simulations and measuring $Z_{m}$ at a fixed frequency we find that the noise powers are distributed around the expected value $Z_{m}=2$ (for one harmonic). If however we consider a range of frequencies and always pick the maximum $Z_{m}$ within that range, it is clear that we will shift the peak of the distribution of noise powers to a higher value. Because of this skewing effect, we choose to use Monte Carlo simulations of the light curve to generate the distribution of fractional amplitudes rather than relying on the theoretical distribution. This issue did not affect the analysis in Section 4.1 because we used a particular frequency model and did not skew the model to maximise $Z_{m}$.

Having established how variable each burst is, we now need take into account the fact that we have a number of bursts. Each burst is an equally valid test of the hypothesis 
that fractional amplitude remains constant during a burst. We therefore need to know the expected distribution of burst variabilities. Fortunately because we are using significances to measure variability the theoretical distribution of variabilities is very simple: the set of values of $V_{r}$ should be uniformly distributed between 0 and 1 . This means that we expect to see a range of burst variability.

The first thing that is clear is that even though we have nearly 30 bursts, Burst 28 constitutes a highly significant outlier. The fractional amplitude for this burst is without doubt varying. The most probable explanation for this is that photospheric radius expansion in the peak of the burst obscures the stellar surface, suppressing the fractional amplitude until touchdown.

What about the first 27 bursts? We will consider these bursts as a set and assume that the physical processes occurring in each burst are the same (Burst 28 is excluded from the set because there is an additional physical process, photospheric radius expansion, at work in this burst). Figure 16 shows that there is a shift in the distribution towards lower values of $V_{r}$, suggesting that there may be some variability. However, we need to evaluate formally the significance of the deviation. There are various ways of doing this but given that the deviation takes the form of a shift, a simple option is to compute the mean of the 27 values of $V_{r}$. We can then use simulations to model the distribution of means (as one wonld expect these values are distributed symmetrically about 0.5 ) and use this distribution to measure the significance of the measured value. We find that the mean of the dataset is 0.420 . However, values smaller than this are obtained in 71 out of 1000 simulations. The hypothesis that fractional amplitude remains constant during the bursts can be rejected at the $1 \sigma$ level but not at a $2 \sigma$ level.

Similar results are obtained if one uses a Kolmogorov-Smirnov (KS) test, a standard technique for measuring deviations from a theoretical distribution. The KS test is supposed to be sensitive to shifts in distribution but should not be used if there are major outliers in the data set (such as Burst 28). The KS test indicates that the probability of obtaining $a$ deviation larger than the observed value and of the same sign as the observed value is 0.12. The hypothesis is again rejected at a $1 \sigma$ level, but not at at $2 \sigma$ level.

In the above analysis we have computed a measure of the variability of each burst and then analysed the distribution of burst variabilities. This method treats each burst as an equally valid test of the hypothesis that fractional amplitude remains constant during a burst. An alternative way of analysing the data is not to treat each burst separately, but to compute a rolling $\bar{\chi}^{2}$ where we sum over all of the 5000 photon data points from all of the bursts. If we do this we find that the simulated $\bar{\chi}^{2}$ exceeds the measured value in 69 simulations out of 1000 , in good agreement with the previous results. Note however that 
by rolling all of the results together we can no longer distinguish between a set of bursts that all have medium variability, and a set where the variability ranges from low to high. The first option would in fact be rather unusual, as it would imply the outliers were evenly distributed throughout the data set, whereas groupings of outliers will occur by chance. We can distinguish this behavior only by treating each burst individually as we did in our initial analysis. Nonetheless, the combined test does (as we would expect) back up our conclusion that we can rule out the constant fractional amplitude hypothesis at a $1 \sigma$ but not a $2 \sigma$ level.

It is possible that the variability could be due to the accretion rather than the burst component, since we have not corrected for accretion in this analysis. If the accretion component is responsible for the variability, one would expect to see higher variability in the lower luminosity bursts where the accretion component plays a larger role. We find no evidence for such a correlation, and conclude that the limited variability that is observed is due to the thermonuclear process.

In calculating fractional amplitude variability, we did not take into account the temporal ordering of data points during a burst. This could obscure interesting effects such as, for example, the peak fractional amplitude always occurring at the peak of the lightcurve. To test for any such effects we proceed as follows. We first convert the fractional amplitude values evaluated during each burst into a percentage shift above or below the burst average value. We then take the full set of data for the first 27 bursts, bin the data points by time elapsed since the start of the burst, and compute the mean percentage shift in fractional amplitude for each time bin. For a range of different time bin sizes, we find no statistically significant deviation from zero: we cannot detect any consistent temporal effect. In particular, we find no evidence for very high fractional amplitudes very early in the burst rise. This effect, seen in the brighter bursts of some LMXBs, is thought to be caused by the rapid spreading of the initial ignition hotspot (Strohmayer, Zhang \& Swank 1997; Strohmayer et al. 1998). Assuming ignition does occur at a point, a similar effect should occur in J1814. Detecting such an effect would however be difficult for two reasons: firstly, the bursts of J1814 are faint; and secondly because the effect would be masked by the accretion-related pulsations.

Let us now move on to consider variability in frequency. The center panels of Figure 14 show dynamical power spectra computed using overlapping $4 \mathrm{~s}$ bins, with new bins being started at intervals of $0.25 \mathrm{~s}$. The lower panels of Figure 14 show the difference between the frequency $\nu_{m}$ at which $Z_{m}$ is maximised and the frequency derived from the best fit orbital model, for non-overlapping $4 \mathrm{~s}$ bins. The error bars on the frequency measurements are derived from simulations, as discussed below.

Monte Carlo simulations show that the difference between the measured peak frequency and the input model frequency depend on both the length of the time bin considered and 
on the value of $Z_{m}$. For this reason we fix the time bin size. The distribution of apparent frequency shifts with $Z_{m}$, for a fixed binsize of $4 \mathrm{~s}$, is shown in Figure 17. It is clear that below a certain value of $Z_{m}$ it is no longer possible to define even a $1 \sigma$ error bar. We therefore set a minimum $Z_{m}$, below which we will not attempt to compute frequency. We set a minimum of $Z_{m}=15$, the lowest level at which we can still compute a $2 \sigma$ error bar. Note that a similar problem afficts phase residuals; below a certain signal strength the uncertainty in the profile fit is sufficiently large that one cannot define error bars on the phase.

The results in the lower panels of Figure 14 are computed using only events that arrive when the flux is at least twice the pre-burst level. We will discuss frequency shifts in the burst rise later in this section. The figures suggest that there is at most only low variability in frequency during the bulk of the burst. Let us now quantify this more precisely. The null hypothesis that we wish to test is that the underlying frequency remains constant, apart from any orbital corrections, during the main part of the burst (excluding the earliest stages of the burst rise). We define the following simple measure of frequency variability:

$$
\bar{\chi}_{\nu}^{2}=\sum_{i}^{N_{\text {bins }}}\left(\nu_{i}-\nu_{\text {model }}\right)^{2}
$$

where $N_{\text {bins }}$ is the number of non-overlapping $4 \mathrm{~s}$ bins in the burst (where flux is at least twice the pre-burst level), $\nu_{i}$ is the $\nu_{m}$ calculated for each bin, and $\nu_{\text {model }}$ is in this case the frequency derived from the orbital ephemeris. The decision to use non-overlapping bins is motivated by the fact that the dynamical power spectra do not show evidence for significant variations in frequency on timescales shorter than $4 \mathrm{~s}$ apart from perhaps in the burst rise phases, which we treat in more detail later in this section.

As before we use Monte Carlo simulations to determine the significance of the $\bar{\chi}_{\nu}^{2}$ measured for each burst. However, the approach that we used previously, simulating the light curve to generate artificial events files, is no longer appropriate. This is because the distribution of frequency shifts depends on $Z_{m}$ and hence on fractional amplitude. We know from the previous section that fractional amplitude may be varying during the bursts; but as we have no model for this variation, we cannot generate simulated lightcurves that would give us an accurate distribution for $\bar{\chi}_{\nu}^{2}$.

Instead we use the simulated distributions of frequency shift as a function of $Z_{m}, f\left(\nu_{m}-\right.$ $\left.\nu_{\text {model }}: Z_{m}\right)$, that were used to generate Figure 17 . For each burst we have a set of $N_{\text {bins }}$ values of $Z_{m}$. If we are running $N_{\mathrm{sim}}$ simulations we therefore start by generating $N_{\mathrm{sim}} \times N_{\text {bins }}$ random numbers drawn from a uniform distribution between 0 and 1 . For each data point in the burst we take $N_{\text {sim }}$ of these $f$ values and read off the corresponding frequency shifts from the simulated cumulative distribution function for the appropriate value of $Z_{m}$. This 
process is repeated for each data point in the burst. We then group the simulated frequency shifts into sets of $N_{\text {bins }}$, each set containing one point from each $Z_{m}$ value, and compute a $\bar{\chi}_{\nu}^{2}$ for that set. For each burst we can then determine the probability $V_{\nu}$ that the simulated $\bar{\chi}_{\nu}^{2}$ exceeds the measured value. This gives us a simple measure of the variability of each burst. The results are given in Table 1 and Figure 18. It is readily apparent that none of the bursts are major outliers in the way that Burst 28 was when we computed $V_{r}$.

As before we now need to take into account the fact that we have 28 bursts. This time we include Burst 28 in our sample because photospheric radius expansion only affects frequency variability in the sense that it suppresses $Z_{m}$, and we have taken this into account in our simulations. The expected distribution of $V_{\nu}$, assuming that there are no shifts beyond orbital corrections, should be uniform. Figure 19 illustrates that there is good agreement between the theoretical distribution and the observed distribution, and certainly no skew towards lower values of $V_{\nu}$, as we would expect if there is significant frequency variability. This is confirmed by the fact that the mean of the set of values of $V_{\nu}$ is 0.530 . A KS test also confrms no significant deviation of the distribution uniformity. In addition if we combine data points from all of the bursts and compute a rolling $\bar{\chi}_{\nu}^{2}$, we find that the measured value is exceeded in 616 out of 1000 simulations. There is therefore no evidence of frequency variability during the main part of the bursts.

We also searched for any statistically significant temporal ordering effects. Such an effect might be the minimum frequency always occurring at the peak of the lightcurve, which could indicate a statistically significant frequency decrease at this time. By combining measurements from all of the different bursts and binning by time elapsed since burst start we have verified that we detect no significant temporal ordering effects in the frequency variability.

The analysis presented thus far has focused on events arriving when the flux is at least twice the pre-burst flux. Let us now consider the burst rise in more detail, by including events that occur before this flux threshold is reached. Strohmayer et al. (2003) noted that for two of the bursts (Bursts 12 and 28; note the difference in burst numbering compared to Strohmayer et al. (2003)), there appeared to be significant frequency decreases during the burst rise. These two apparent shifts, visible in Figure 14, are sufficiently rapid that we must use either overlapping time bins or shorter time bins to resolve them.

The difficulty with measuring frequency shifts in the burst rise is that in general, the signal $\left(Z_{m}\right)$ is weak, which can lead to large statistical variations. If we use shorter, say $2 \mathrm{~s}$ time bins, this increases the statistical scatter of the frequency measurement, particularly for weak signals (effectively broadening the error bars shown in Figure 17). Overlapping time bins also have their problems, however, as they give rise to correlated noise. 
The dynamical power spectrum and phase residual evolution for the rise of Burst 12 are shown in Figure 20. The power spectrum suggests a frequency very close to the orbital frequency before the burst. The frequency then jumps a few tenths of a $\mathrm{Hz}$ above the orbital frequency and gradually slides back down to the orbital frequency before the burst rise is complete. We must determine whether the jump and subsequent slower drop in the frequency are statistically significant. We will argue that they are not.

The first point after the jump has $Z_{m} \approx 10$. For such a weak signal obtaining an apparent frequency shift of the observed magnitude or larger is possible in $\approx 10 \%$ of simulations. Given that we have 28 bursts, therefore, this is not unusual. The events that gave rise to the initial large shift are still present in the next 4 seconds' worth of power spectra. Could they be acting to keep the frequency high, given that by the next independent time bin the frequency is back to the orbital frequency? The answer is yes provided that the frequency shift is sufficiently large and the light curve climbs only slowly over the period. Both of these conditions are met in this burst.

To verify whether this is the case we have re-computed the dynamical power spectra using shorter $(2 \mathrm{~s})$ bins, to reduce correlation effects. Although there is still some movement in frequency the large downshift is no longer apparent. The magnitude of the shift is not statistically significant given our sample size. Dynamical power spectra and the associated phase residuals for the $2 s$ bins are shown in Figure 20.

The dynamical power spectrum and phase residual evolution for the rise of Burst 28 are shown in Figure 21. The peak frequency shifts from the orbital frequency down by $\approx 0.15 \mathrm{~Hz}$ during the burst rise. The peak signal during this phase is strong: in the range $Z_{m}=60-80$. For such a strong signal correlated noise is unlikely to play a role, and we have confirmed this by re-computing the dynamical power spectrum using shorter time bins. The shift is also readily apparent from the phase residuals. Simulations indicate that the probability of obtaining a frequency shift this large for a signal this strong is less than $1 \mathrm{in} 10^{4}$. Even given the number of bursis sampled this is a significant outlier. We can with a high degree of confidence assert that the frequency drops below the orbital frequency in the rise of Burst 28.

In summary, none of the bursts apart from Burst 28 show any evidence for frequency shifts in the burst rise. Burst 28 , by contrast, shows an extremely signincant drop in frequency of approximately $0.1 \mathrm{~Hz}(0.05 \%)$ over a timescale $\sim 1 \mathrm{~s}$. The significance of this result is discussed in the next section. 


\section{Discussion and conclusions}

Our aim in analysing the variability of the burst oscillations of J1814 was to constrain models of the burst oscillation mechanism in accreting millisecond pulsars. We have focused on three aspects of the oscillations: fractional amplitude, harmonic content, and frequency. The first two properties constrain the size, shape and position of the emitting region, whilst changes in frequency indicate motion of the emitting region.

We began by considering variations in the average burst properties over the course of the outburst, with the goal of teasing out the influence of the accretion and burning history. In the second part of the study we focused on the short-term variability during the bursts, as a direct probe of the progression of the thermonuclear process. Our conclusions are as follows.

We can rule out the hypothesis that burst average fractional amplitude remains constant at a level greater than 1 in $10^{4}$. We can also rule out, at a similar level, the hypothesis that the burst fractional amplitude for all of the bursts is the same as the daily average non-burst fractional amplitude. The problem is in fact rather complex.

For most of the bursts the average fractional amplitude is in good agreement with the accretion fractional amplitude. Since accretion fractional amplitude reflects the pattern of fuel deposition on the stellar surface, this implies a low degree of fuel spread. A factor that could restrict spreading is magnetic confinement, but the viscosity of the accreted fuel will also be important. If this is the case then the hotspot size, position and inclination inferred by Bhattacharyya et al. (2005) from the burst oscillations should hold true for the accretion hotspot. The geometry inferred by Bhattacharyya et al. (2005) is that illustrated in the center panel of Figure 6.

There are however 6 bursts where the fractional amplitude is substantially lower than the non-burst fractional amplitude, implying a higher degree of fuel spread. The lowest fractional amplitude burst is the final one, which takes place in a lower accretion rate regime. This burst shows the hallmarks of photospheric radius expansion, which would suppress fractional amplitude. However even if we neglect events from the peak of the burst (where the signal is suppressed) the fractional amplitude remains below the non-burst level. At lower accretion rates recurrence times should be longer, perhaps allowing a higher degree of fuel spread between bursts. For the remaining 5 bursts, however, it is difficult to understand why only a subset of the bursts are affected if the only factor affecting spread is the time elapsed since the last burst. One possibility is that the explosive burning front of the previous burst has forced fuel out across the stellar surface. In this case the full burst history, particularly the nature of the precursor burst, becomes important. It is however difficult to track this given 
the gaps in the RXTE coverage.

J1814 shows the strongest first harmonic content of the known accreting millisecond pulsars, so it forms an interesting case for analysis: We find that the fractional amplitude of the first harmonic is in general lower for the bursts than for the non-burst pulsations. We can in fact rule out the hypothesis that the first harmonic content is the same as that of the non-burst emission with a high degree of confidence (at a level greater than 1 in $10^{4}$ ). We cannot however rule out the hypothesis that the fractional amplitude of the first harmonic during the bursts remains constant over the course of the outburst for the 27 bursts that do not exhibit photospheric radius expansion.

This apparent difference in behavior between the fundamental and first harmonic is interesting. If first harmonic content is determined only by surface emission region geometry then the discrepancy in first harmonic content of the burst and non-burst emission is hard to explain, given that the results for the fundamental suggest that the emission regions are very similar $\frac{f}{1}$ or most of the bursts. One possible explanation is that the first harmonic content of the accretion-related pulsations is boosted by a contribution from boundary layer processes. Boundary layer emission is not apparent during the bursts, when thermonuclear emission from the surface dominates. This explanation would be consistent with results for J1808; Gilfanov et al. (1998); Gierliński, Done \& Barret (2002); Poutanen \& Gierliński (2003) conclude that the first harmonic content of the non-burst pulsations of J1808 is due to Comptonized emission from the boundary layer. One could test this hypothesis by carrying out spectroscopic studies similar to those undertaken for J1808; we leave this as a topic for future work. It is also interesting to note that the first harmonic content of the non-burst emission seems to drop over the course of the outburst. One possible explanation for this effect is magnetic field burial caused by accretion.

The previous study by Strohmayer et al. (2003) noted apparent variations in fractional amplitude of up to $5 \%$ during the course of the bursts. If genuine, such rariations would signal rapid and rather large changes in the thermal patterns on the stellar surface, something that is rather difficult to explain. On the basis of the more detailed analysis presented in this paper we can draw several conclusions regarding variability.

The first is that Burst 28, the final burst, shows compelling evidence for variation in fractional amplitude. We can rule out the hypothesis that the fractional amplitude remains constant at a level greater than 1 in $10^{4}$ for this burst. The nature of the variation is that fractional amplitude is strongly suppressed in the peak of the lightcurve. This is strong evidence for photospheric radius expansion in this burst.

For the remaining 27 bursts, where there is no evidence for photospheric radius ex- 
pansion, we can rule out the hypothesis that fractional amplitude remains constant during a burst at a $1 \sigma$ but not at a $2 \sigma$ level. This is not a particularly strong rejection of the hypothesis. It does indicate that there may be some variability, but underlying variations at a level of $<1 \%$ should be sufficient to explain the variations observed in the data. This result simplifies the modelling requirement, as there is no need to invoke large fluctuations in hotspot area or brightness to explain the data. The variations that are present appear to be random and not dependent on progress through the burst; we detect no sign of any consistent time-dependent effects.

We note that variations in fractional amplitude of similar magnitude have been observed during the bursts of several non-pulsing LMXBs (Muno, Özel \& Chakrabarty 2002). Our analysis shows that statistical scatter alone can lead to apparent variations in fractional amplitude, particularly if one has a large sample of bursts. The only reliable way to assess the significance of the observed variations is to undertake the type of analysis that we have done for J1814. It would be interesting to perform a similar study for the bursts of the LMXBs, particularly since the mechanism behind the asymmetries may differ from that operating in the pulsars.

Some LMXBs show significant fractional amplitude decreases in the burst rise. The fractional amplitude shifts are thought to be the hallmark of the spreading ignition hotspot (Strohmayer, Zhang \& Swank 1997; Strohmayer et al. 1998). We find no evidence for fractional amplitude decrease in the burst rise, but caution that such an effect could be masked by the accretion pulsations in this source.

The bursis of many LMXBs show evidence for frequency shifts during the burst tail, suggesting motion of the thermal patterns on the surface. Our analysis confirms the earlier result of Strohmayer et al. (2003), that the bursts of J1814 show no evidence for frequency shifts in the burst tail, beyond that expected due to orbital Doppler shifts. The data are therefore consistent with the concept that the pulsed burst emission is due to the presence of a hotspot that is near stationary in the rotating frame of the neutron star, most likely at. the visible magnetic polar cap.

We also looked for evidence of frequency shifts in the burst rise. None of the bursts apart from the final one show evidence of a statistically significant frequency shift in the burst rise. The final burst, however, exhibits a small $(\approx 0.1 \mathrm{~Hz})$ but highly significant frequency decrease during the burst rise.

A simple mechanism that could lead to a frequency decrease is expansion of the burning layers. If the layers decouple even partially from the underlying star their rotation rate will drop due to conservation of angular momentum (Strohmayer et al. 1997). The fact that the 
shift is so small, however, implies either a low degree of expansion ( $\sim 1 \mathrm{~m}$ ) or partial coupling, perhaps due to the magnetic field. The fact that the final burst is the most luminous would explain why the heating and expansion is greatest in this burst.

The expansion model has also been suggested as an explanation for the frequency shifts seen in some LMXB bursts (although it does have limitations, see for example Cumming et al (2002)). In a number of LMXB bursts the frequency is observed to rise from a low point at the peak of the lightcurve towards an asymptotic frequency in the burst tail (see for example Muno et al (2002)). The rise is attributed to the cooling contracting burning layer recoupling with the underlying star and hence spinning up. The fact that the frequency decrease is not seen has been attributed to the fact that the timescale on which the burning layers first expand is very short $\left(\sim 10^{-6} \mathrm{~s}\right)$ compared to the time on which photons diffuse through the atmosphere (Cumming \& Bildsten 2000).

The timescale of the frequency shift in the rise of Burst 28 is $\sim 1 \mathrm{~s}$, far slower than the hydrostatic expansion timescale. If hydrostatic expansion of the burning layers is responsible, some mechanism must be acting to slow the expansion. Although it is conceivable that the magnetic field could act in this way, the difference in the timescales is substantial and it seems unlikely that the field could have such a large effect. Another possibility is that the expansion time is slower, perhaps due to a larger amount of hydrogen in the burning mix compared to the LMXB bursts in which oscillations are seen. Expansion time could also be slower if the outer layers of the atmosphere, which expand more slowly, play a more significant role in the emission. A second possibility is that the burning layers expand primarily laterally rather than radially, with only a limited amount of radial expansion as the layers heat up. Lateral expansion is plausible in a situation where the fuel is confined to an elevated blister at the magnetic polar caps rather than being distributed symmetrically. It could also explain the drop in fractional amplitude towards the burst rise, something that has previously been attributed to photospheric radius expansion. There is however no evidence for lateral expansion in any of the other bursts studied. The final possibility, of course, is that we are observing something other than burning layer expansion.

In addition to the issues discussed above, there are several other areas that merit further study. One area that we were not able to address in much detail was the influence of the accretion and burning history over the course of the outburst. The level of coverage achieved by RXTE dropped severely in the second half of the outburst, leading to a much reduced number of burst detections. This is unfortunate, since the only burst detected in the final portion of the outburst (when accretion rate had dropped dramatically) seemed to differ greatly from the other bursts. Without more examples of bursts in the later phase of the outburst, however, it is difficult to determine to whether there is a major change in behavior 
at late times. If J1814 goes into outburst again it would be highly desirable to get more even coverage throughout the outburst.

A second question concerns the non-burst pulsations. We have discussed the relationship between the burst and non-burst pulsations, pointing out both similarities and important differences. However we have as yet little understanding of the factors that control variations in the fractional amplitude and harmonic content of the non-burst pulsations.

We have also been unable to shed much light on the observed variability in burst flux; in particular on the nature of the lowest luminosity bursts. There are hints from the data that the low luminosity bursts may have lower average fractional amplitudes than most of the bursts. However, without a larger sample it is not possible determine whether this is a statistically robust result.

We finish on a cautionary note. Our analysis has shown that the data are consistent with there being only limited variability in fractional amplitude over the course of a burst. Unfortunately we have as yet no models of the predicted variability to test. However, a similar analysis could easily be performed should such a model be developed. Our study also illustrates the need for high PCU coverage. J1814 is a relatively faint burst source, and we are trying to measure short-timescale variations in quantities that vary widely due to statistical effects alone. Most of the bursts in the 2003 outburst were observed with only 3 PCUs. Increasing the number of PCUs for future observations of this source would allow far more stringent tests of the hypotheses examined in this paper.

This research has made use of data obtained from the High Energy Astrophysics Science Archive Research Center (HEASARC) provided by NASA's Goddard Space Flight Center.

\section{REFERENCES}

Basko M.M. \& Sunyaev R.A., 1976, MNRAS, 175, 395

Bhattacharyya S., Strohmayer T.E., Miller M.C., Markwardt C.B., 2005, ApJ, 619, 48.3

Buccheri R., Bennett K., Bignami G.F, Bloemen J.B.G.M., Boriakoff V., Caraveo P.A., Hermsen W., Kanbach G., Manchester R.N., Masnou J.L., Mayer-Hasselwander H.A., Özel M.E., Paul J.A., Sacco B., Scarsi L., Strong A.W., 1983, A\&A, 128, 245

Chakrabarty D., Morgan E.H., 1998, Nature, 394, 346 
Chakrabarty D., Morgan E.H., Muno M.P., Galloway D.K., Wijnands R., van der Klis M., Markwardt C.B., 2003, Nature, 424, 42

Cui W., Morgan E.H., Titarchuk L., 1998, ApJ, 504, L27

Cumming A., Bildsten L., 2000, ApJ, 544, 453

Cumming A., Zweibel E., Bildsten L., 2001, ApJ, 557, 958

Cumming A., Morsink S.M., Bildsten L., Friedman J.L., Holz D.E., 2002, ApJ, 564, 343

Done C., Gierliński M., 2003, MNRAS, 342, 1041

Fujimoto M.Y., Hanawa T., Miyaji S., 1981, ApJ, 247, 267

Fushiki I., Lamb D.Q., 1987, ApJ, 323, L55

Galloway D.K., Chakrabarty D., Morgan E.H., Remillard R.A., 2002, ApJ, 576, L137

Galloway D.K., Cumming A., Chakrabarty D., 2004, AAS/HEAD meeting 25.01

Galloway D.K., Markwardt C.B., Morgan E.H., Chakrabarty D., Strohmayer T.E., 2005, ApJ, 622, L 4.5

Ghosh P., Lamb F.K., 1979, ApJ, 232, 259

Gierlińshi M., Done C., Barret D., 2002, MNRAS, 331, 141

Gilfanov M., Revnivtsev M., Sunyaev R.A., Churazov E., 1998, A\&A, 338, L83

Groth E.J., 1975, ApJS, 29, 285

Heyl J., 2004, ApJ, 600, 939

Inogamov N.A., Sunyaev R.A., 1999, Astron. Letters, 25, 269

Krauss M.I., Wang Z., Dullighan A., Juett A.M., Kaplan D., Chakrabarty D., van Kerkwijk M.H., Steeghs D., Jonker P., Markwardt C.B., 2005, astro-ph/0503671

Leahy D.A., Darbro W., Elsner R.F., Weisskopf M.C., Kahn S., Sutherland P.G., Grindlay J.E., 1983, ApJS, 266, 160

Lee U., 2004, ApJ, 600, 914

Lee U., Strohmayer T.E., 2005, astro-ph/0502502 
Lyubarskii Y.E., Sunyaev R.A., 1982, SvAL, 8, 612

Markwardt C.B., Swank J.H., Strohmayer T.E., in't Zand J.J.M., Marshall F.E., 2002, ApJ, $575, \mathrm{~L} 21$

Markwardt C.B., Smith E., Swank J.H., 2003, IAU Circ., 8080

Markwardt C.B., Swank J.H., 2003, IAU Circ., 8144

McDermott P.N., Taam R.E., 1987, ApJ, 318, 278

Miller M.C., Lamb F.K., Psaltis D., 1998, ApJ, 508, 791

Muno M.P., Chakrabarty D., Galloway D.K., Psaltis D., 2002, ApJ, 580, 1048

Muno M.P., Özel F., Chakrabarty D., 2002, Ap.J, 581, 550

Piro A.L., Bildsten L., 2005, astro-ph/0502.546

Poutanen J., Gierliński M., 2003, MNRAS, 343, 1301

Press W.H., Flannery B.P., Teukolsky S.A., Vetterling W.T., 1992, Numerical Recipes in Fortran', Cambridge University Press

Scharlemann E.T., 1978, Ap.J, 219, 617

Spitkorsky A., Levin Y., Ushomirsky G., 2001, Ap.J, 566, 1018

Spruit H.C., Taam R.E., 1990, A\&A, 229, 475

Strohmayer T.E., Markwardt C.B., 2002, Ap.J, 581, 577

Strohmayer T.E., Bildsten L., 2005, to appear in 'Compact Stellar X-Ray Sources', eds. Lewin W.H.G. \& van der Klis M., Cambridge University Press

Strohmayer T.E., Zhang W., Swank J.H., 1997, Ap.J, 487, L77

Strohmayer T.E., Jahoda K., Giles A.B., Lee U., 1997, ApJ, 486, 355

Strohmayer T.E., Zhang W., Swank J.H., White N.E., Lapidus I., 1998, ApJ, 498, L135

Strohmayer T.E., Markwardt C.B., Swank J.H., in't Zand J.J.M, 2003, ApJ, 596, L67

Taam R.E., 1980, Ap.J, 241, 358 
van der Klis M., 199.5, in 'X-Ray Binaries', eds. Lewin W.H.G., van Paradijs J. \& van den Heuvel E.P.J., Cambridge University Press, p.252

van Straaten S., van der Klis M., Wijnands R., 2005, ApJ, 455, 482

Vaughan B.A., van der Klis M., Wood K.S., Norris J.P., Hertz P., Michelson P.F., van Paradijs J., Lewin W.H.G., Mitsuda K., Penninx W., 1994, ApJ, 435, 362

Wall J.V., Jenkins C.R., 2003, 'Practical Statistics for Astronomers', Cambridge University Press

Wijnands R., van der Klis M., 1998, Nature, 394, 344

Woosley S.E., Heger A., Cumming A., Hoffman R.D., Pruet J., Rauscher T., Fisker J.L., Schatz H., Brown B.A., Wiescher M., 2004, ApJS, 151, 75 


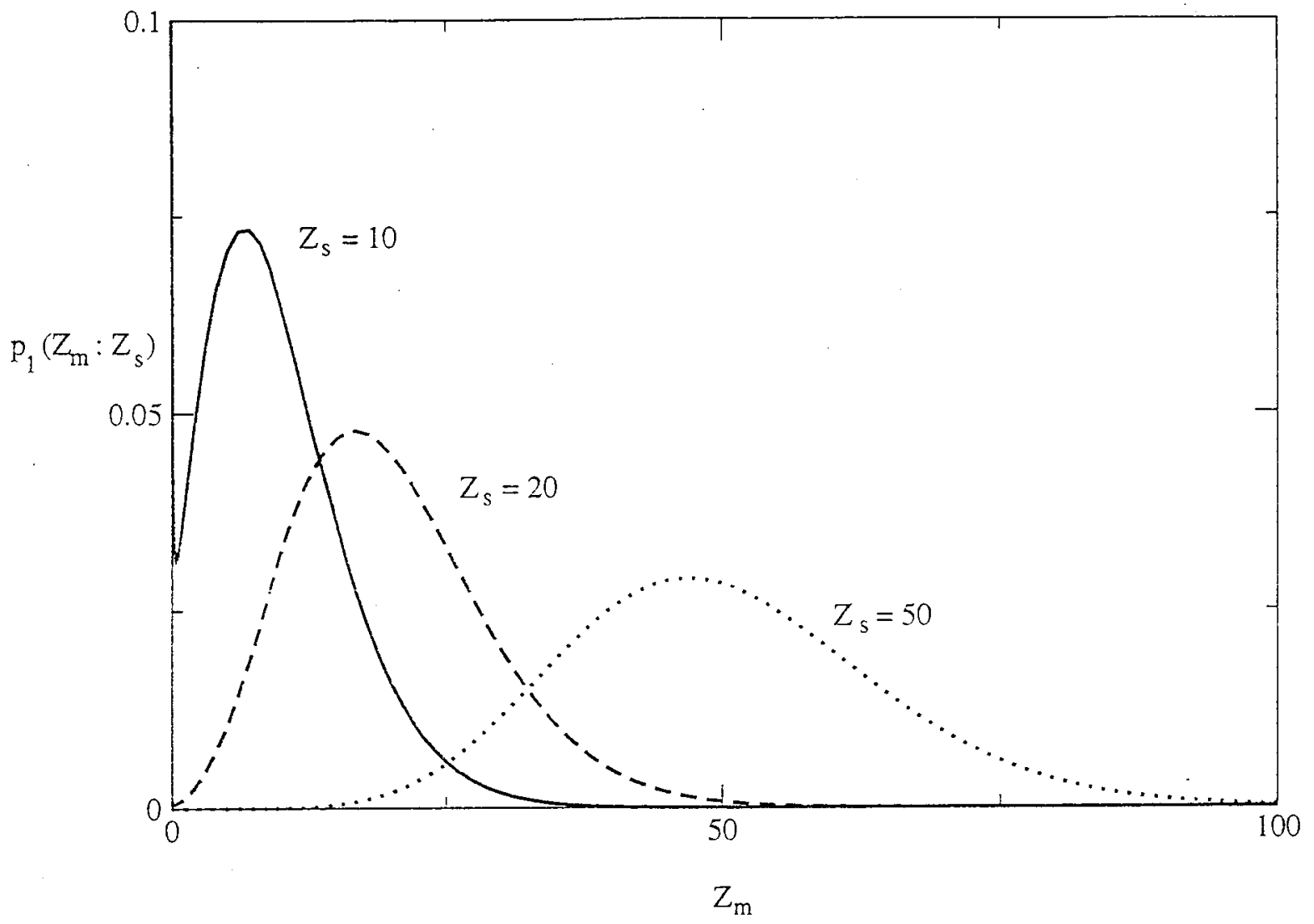

Fig. 1.- Probability distribution $p_{n}\left(Z_{m}: Z_{s}\right)$ (equation 6), for 1 harmonic $(n=1)$, showing the effect of varying signal power $Z_{s}$. The distribution deviates significantly from a normal distribution for low $Z_{s}$. 

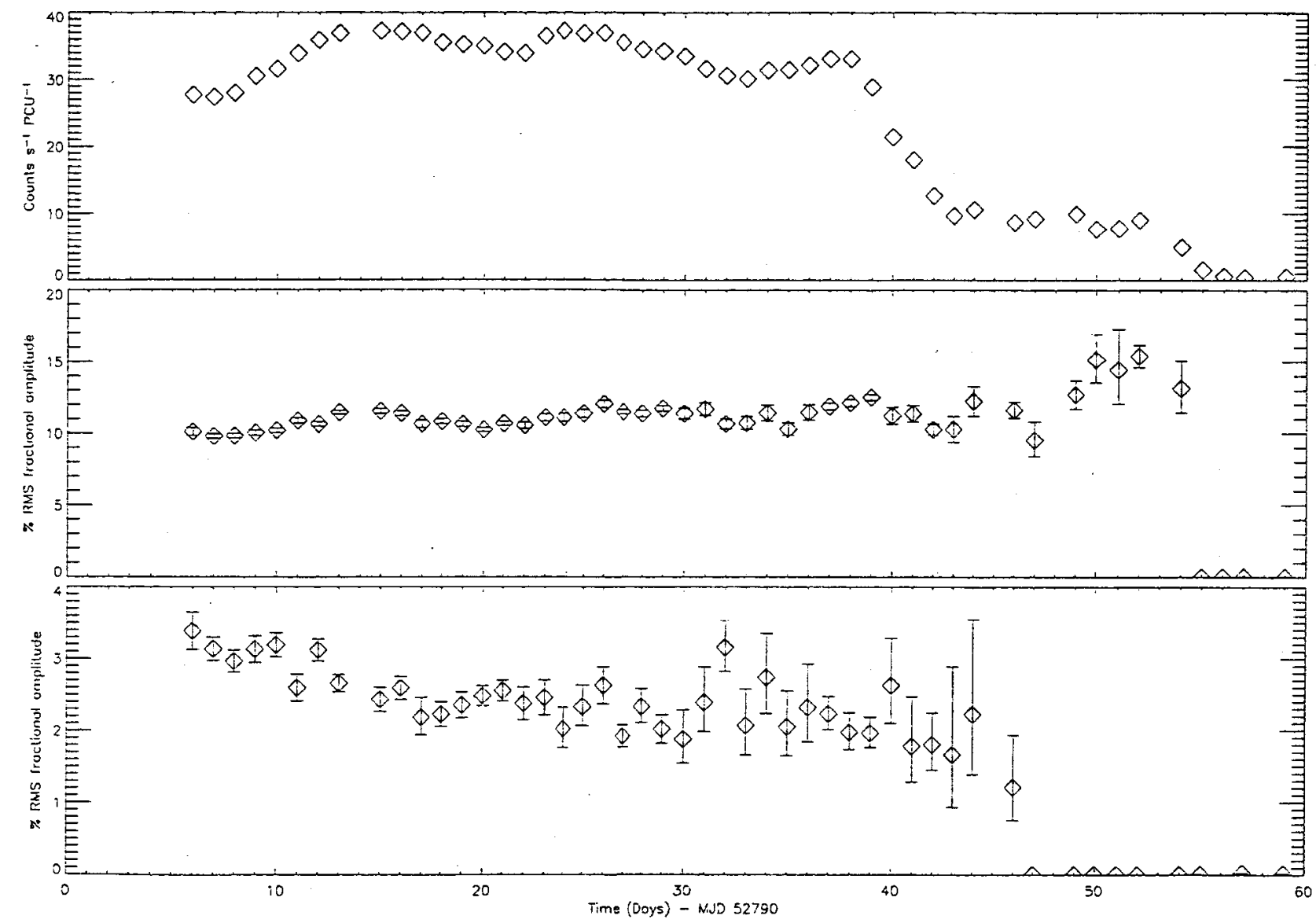

Fig. 2.- Upper panel: Daily average countrate in the energy band $2.5-25 \mathrm{keV}$, corrected for background. Center panel: Daily average RMS fractional amplitude (\%) at the fundamental frequency of the pulsar. Lower panel: Daily average RMS fractional amplitude (\%) at the inst harmonic of the pulsar frequency. Where fractional amplitudes drop to zero the signal can no longer be detected at even a $1 \sigma$ level. 

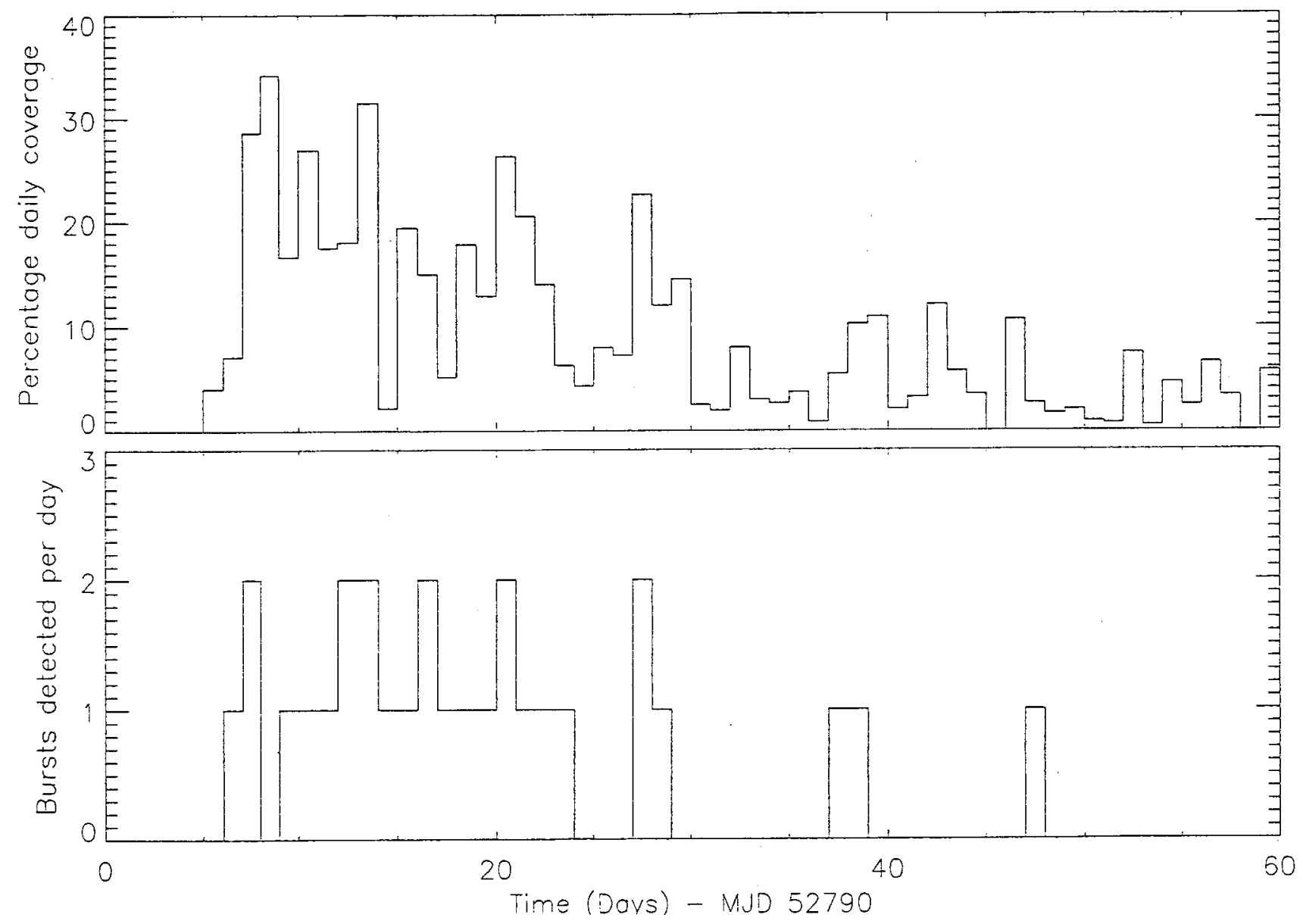

Fig. 3. - The upper panel shows the percentage daily coverage achieved by the RXTE PCA during the 2003 outburst. The lower panel shows the number of bursts detected per day. 


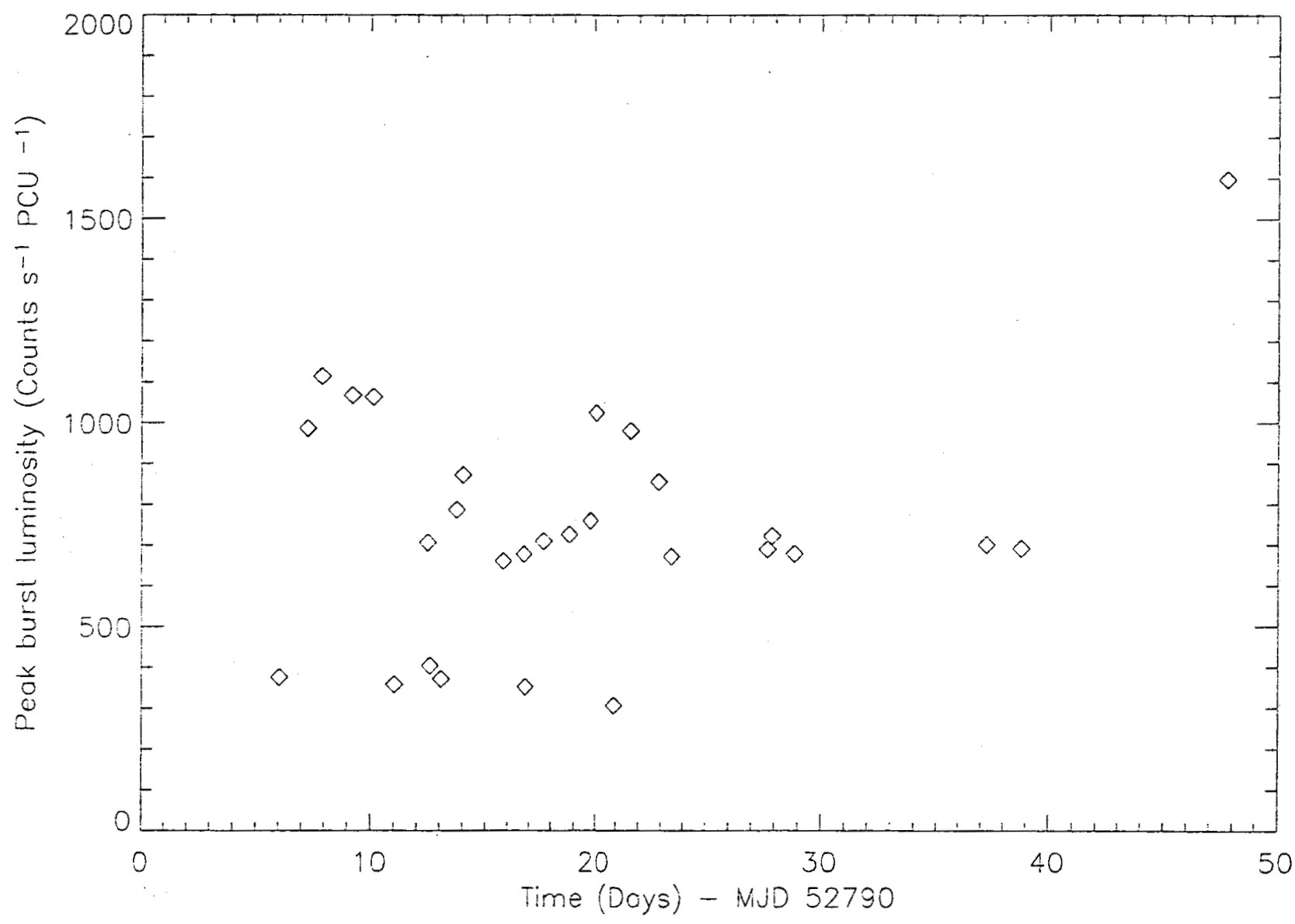

Fig. 4. - The evolution of peak burst count rate (in the energy band $2.5-25 \mathrm{keV}$, corrected for background and persistent emission) through the outburst. 


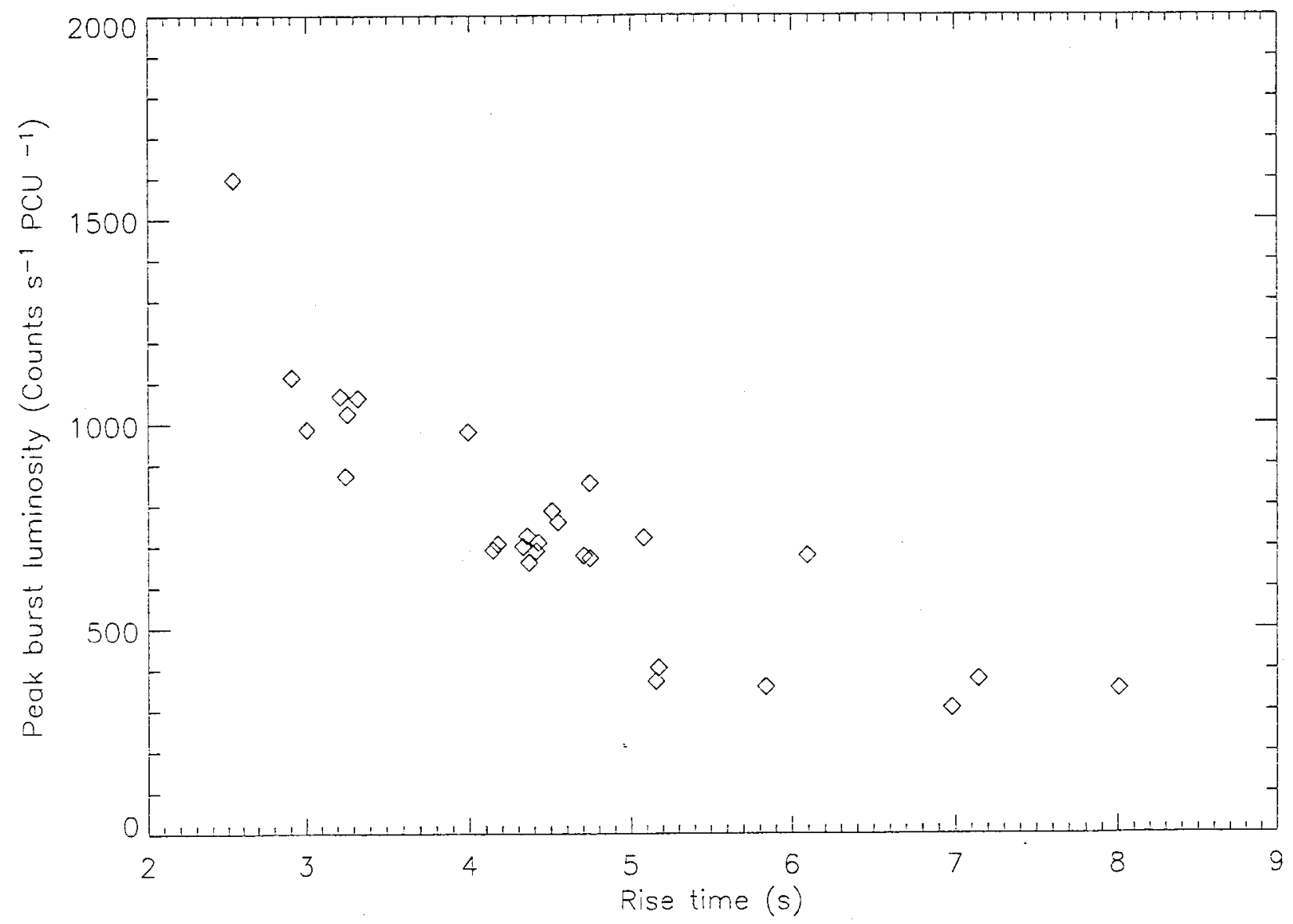

Fig. 5.- Peak burst count rate (in the energy band $2.5-25 \mathrm{keV}$, corrected for background and persistent emission) against burst rise time. Rise time is defined as the time it takes the corrected count rate to rise from $10 \%$ to $90 \%$ of the peak value. 

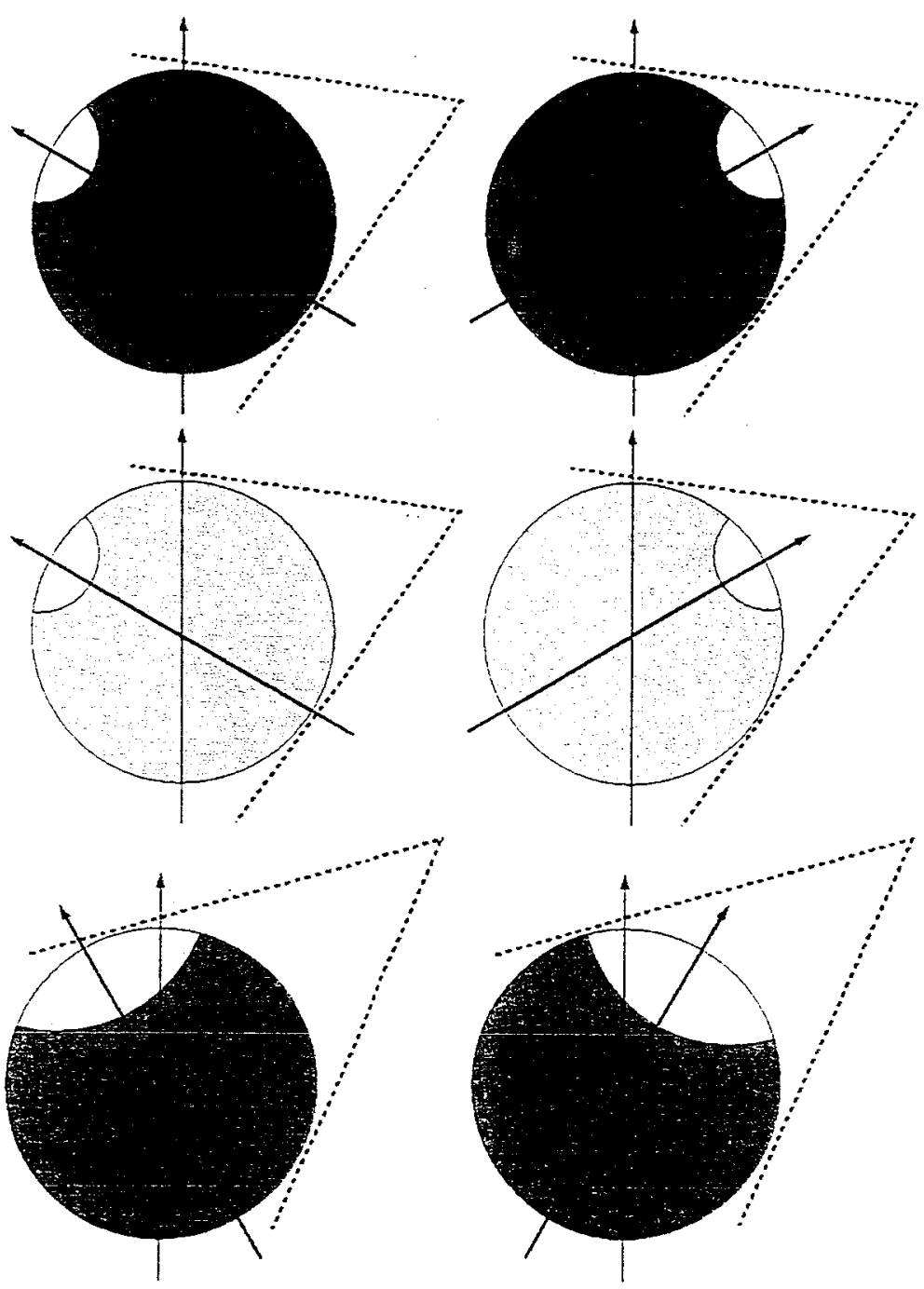

Fig. 6. - The effects of different geometries and degrees of magnetic channeling on nonburst pulsation amplitude. The dashed lines represent the field of view of the observer. The vertical arrow marks the rotation axis, the inclined arrow the magnetic dipole axis. The white area is the visible magnetic polar cap (the antipodal cap is not shown). The rest of the star is shaded either dark or light grey depending on its luminosity. The left and right panes in each of the three panels indicate how the observer's view changes as the pulsar rotates. In the upper panel, magnetic channeling is strong. The bulk of the material falls onto the magnetic polar caps, giving a high luminosity in this region. The rest of the star has very low luminosity. The geometry is such that the hot magnetic cap rotates out of the field of view, giving a high fractional amplitude. In the center panel, the geometry remains the same, with the spot rotating out of the field of view. This time however, magnetic channeling is weaker and the rest of the star is correspondingly brighter. Fractional amplitude will be lower. In the lower panel magnetic channeling is again strong, but this time the geometry (spot size and observer inclination) is such that some portion of the hot cap is always in view. Fractional amplitude will again be lower. 

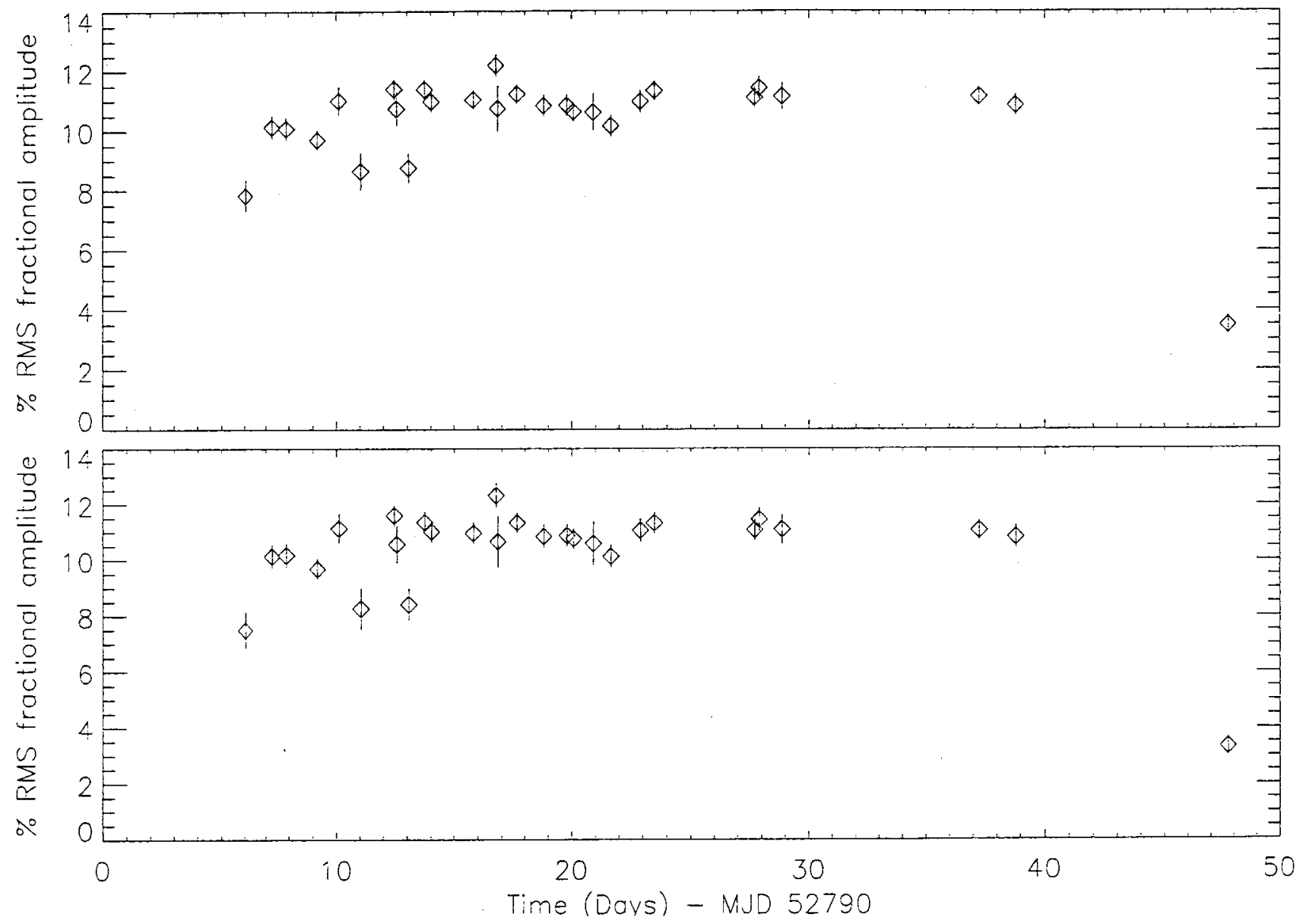

Fig. 7.- Burst average fractional amplitude at the fundamental frequency of the oscillations, showing variation over the course of the outburst. The fractional amplitude is calculated using only events arriving between points when the flux is at least twice the pre-burst level. The values in the upper panel are not corrected for accretion; the lower values are corrected. The effect of including the accretion correction is for the most part imperceptible; the largest changes (a reduction of 4-5\% of the uncorrected value) are to Bursts 1, 6, 9 and 28. 

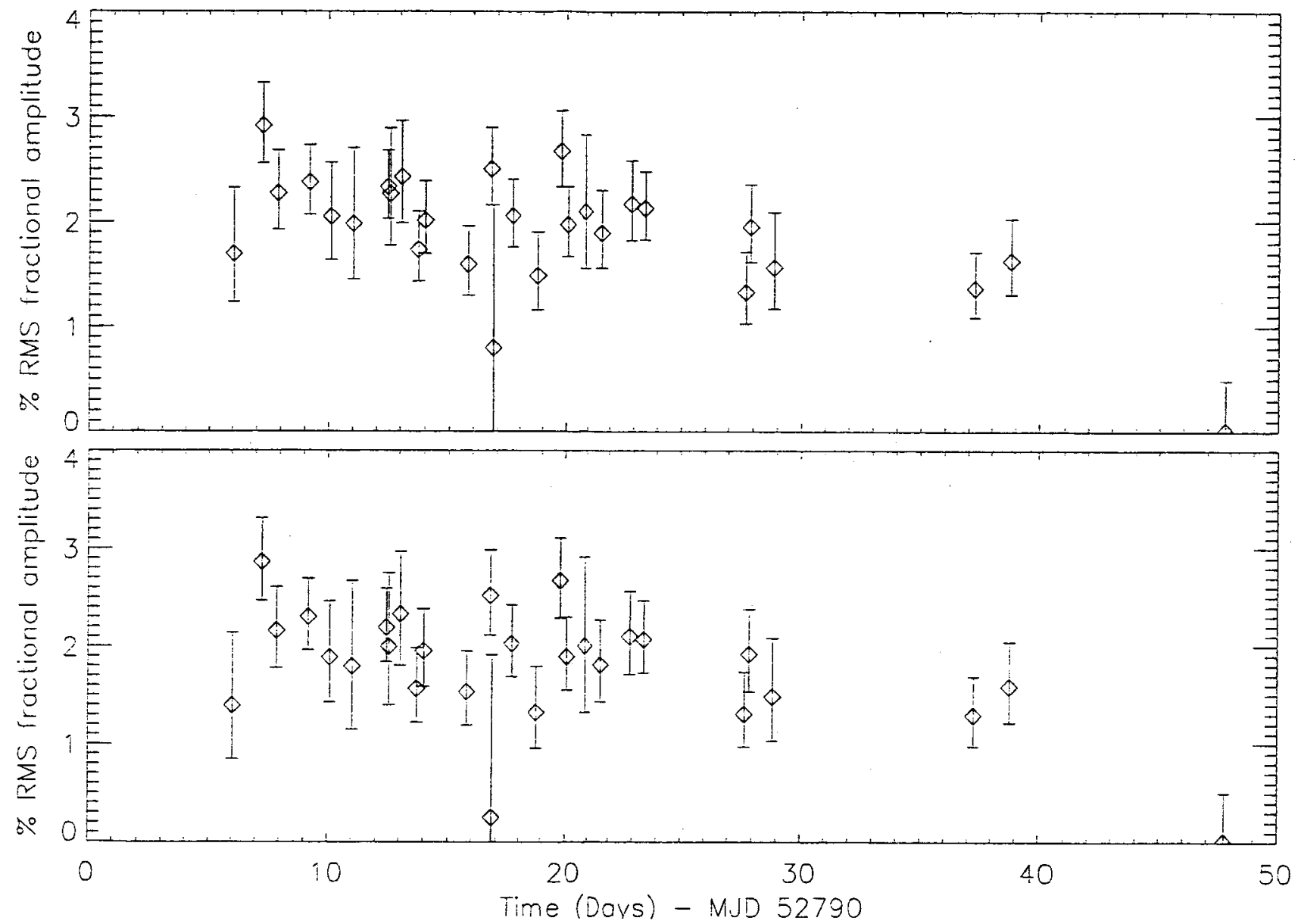

Fig. 8.- Burst average fractional amplitude at the first harmonic of the oscillations, showing variation over the course of the outburst. The fractional amplitude is calculated using only events arriving between points when the flux is at least twice the pre-burst level. The values in the upper panel are not corrected for accretion; the lower values are corrected. In this case the effect of including the accretion contribution is noticeable. For ten bursts $(1,5,6,7$, $8,10,14,16,25$ and 26) the fractional amplitude drops by more than $5 \%$ of the uncorrected value. 


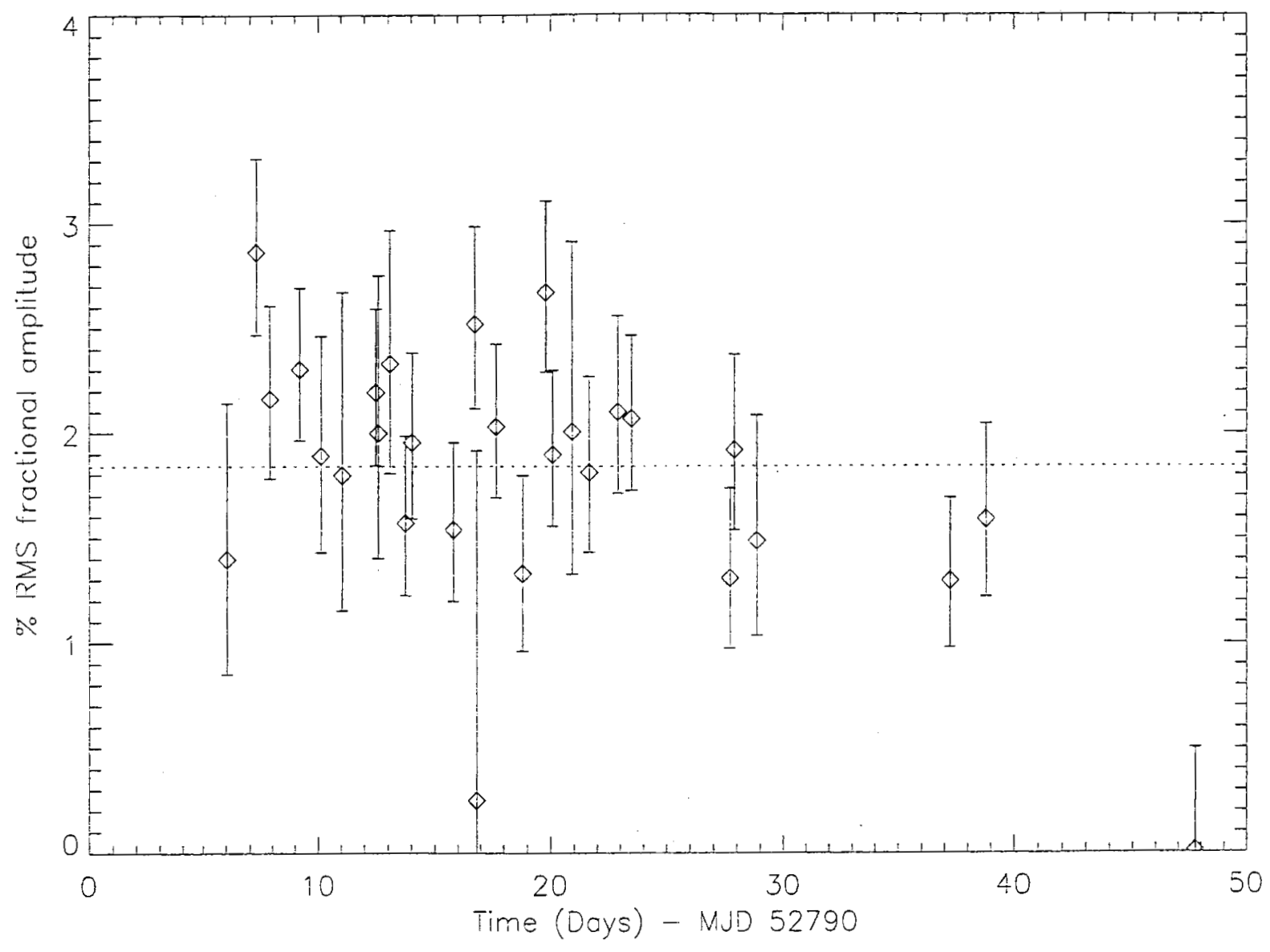

Fig. 9.- Best fit constant fractional amplitude at the first harmonic of the burst oscillation frequency for the first 27 bursts. The measured fractional amplitudes shown are accretioncorrected values. 


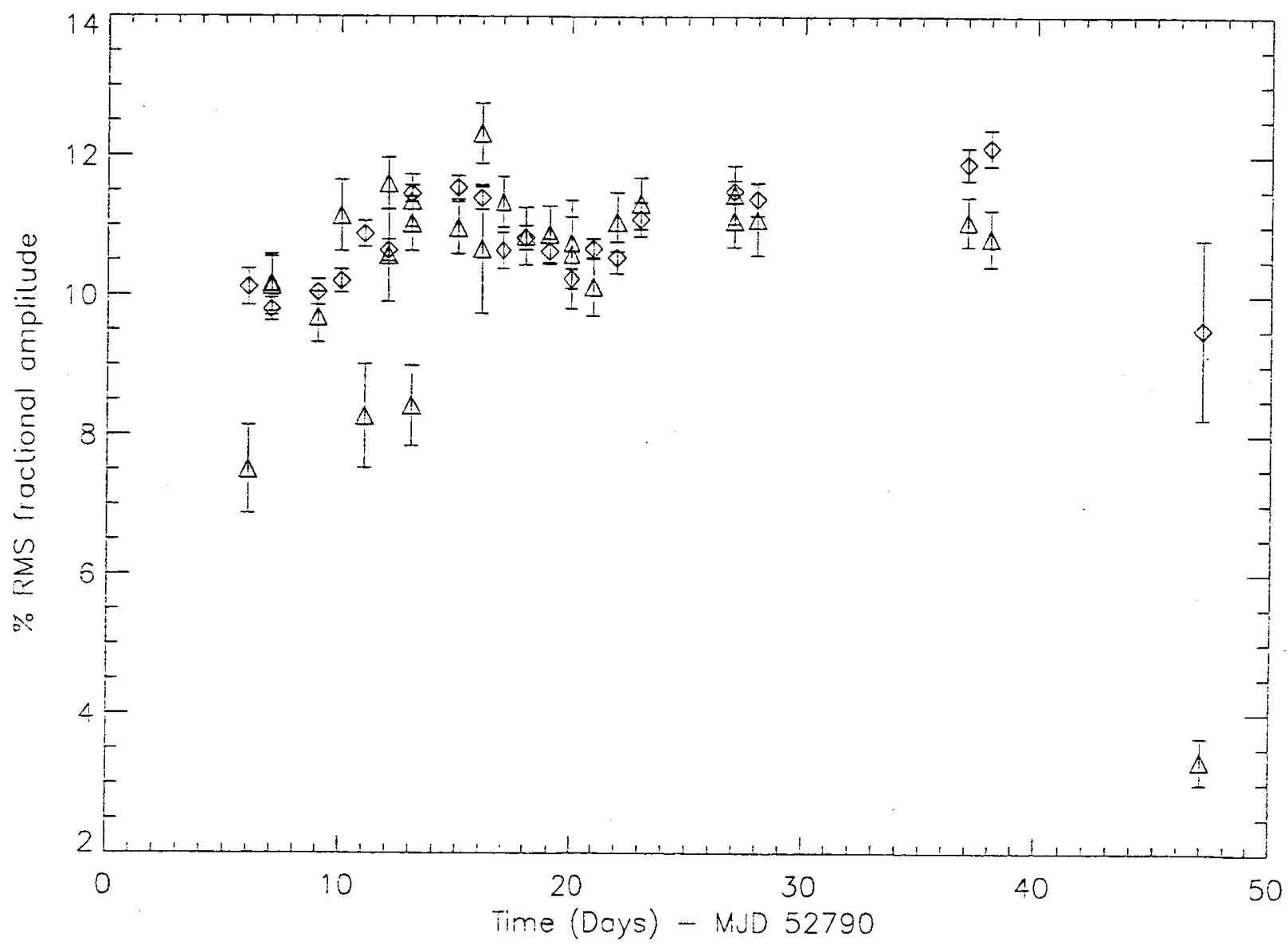

Fig. 10.- The diamonds mark the non-burst daily average fractional amplitude of the fundamental on the days when bursts occur; the triangles the burst average fractional amplitude. For most of the bursts there is relatively good agreement between burst and non-burst values, but for Bursts 1, 6, 9, 26, 27 and 28 the fractional amplitude of the bursts is far lower than that of the non-burst pulsations. 


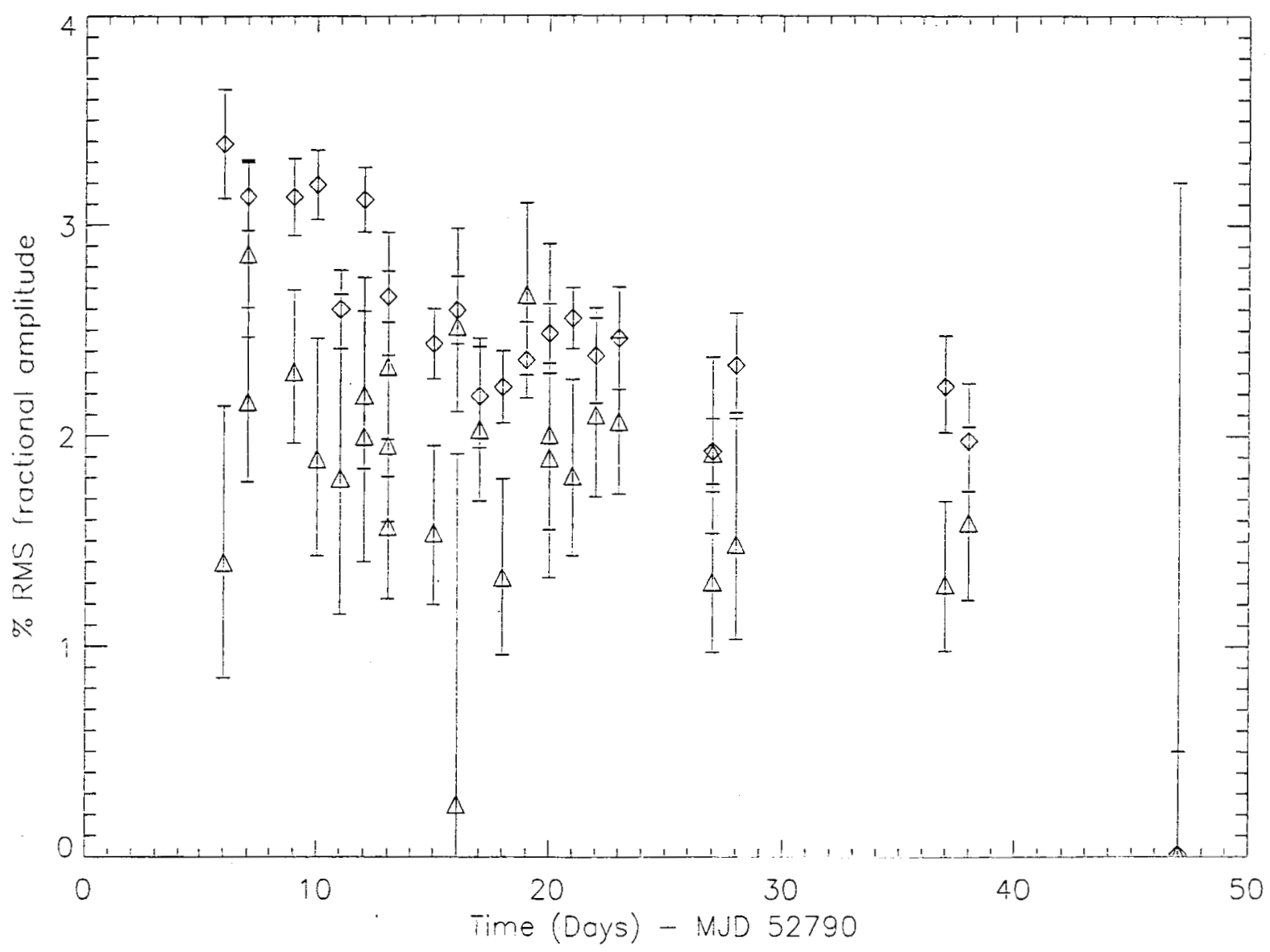

Fig. 11. - The diamonds mark the non-burst daily average fractional amplitude of the first harmonic, the triangles the burst average fractional amplitude. The accretion-corrected burst fractional amplitudes are consistently lower than the accretion values. 

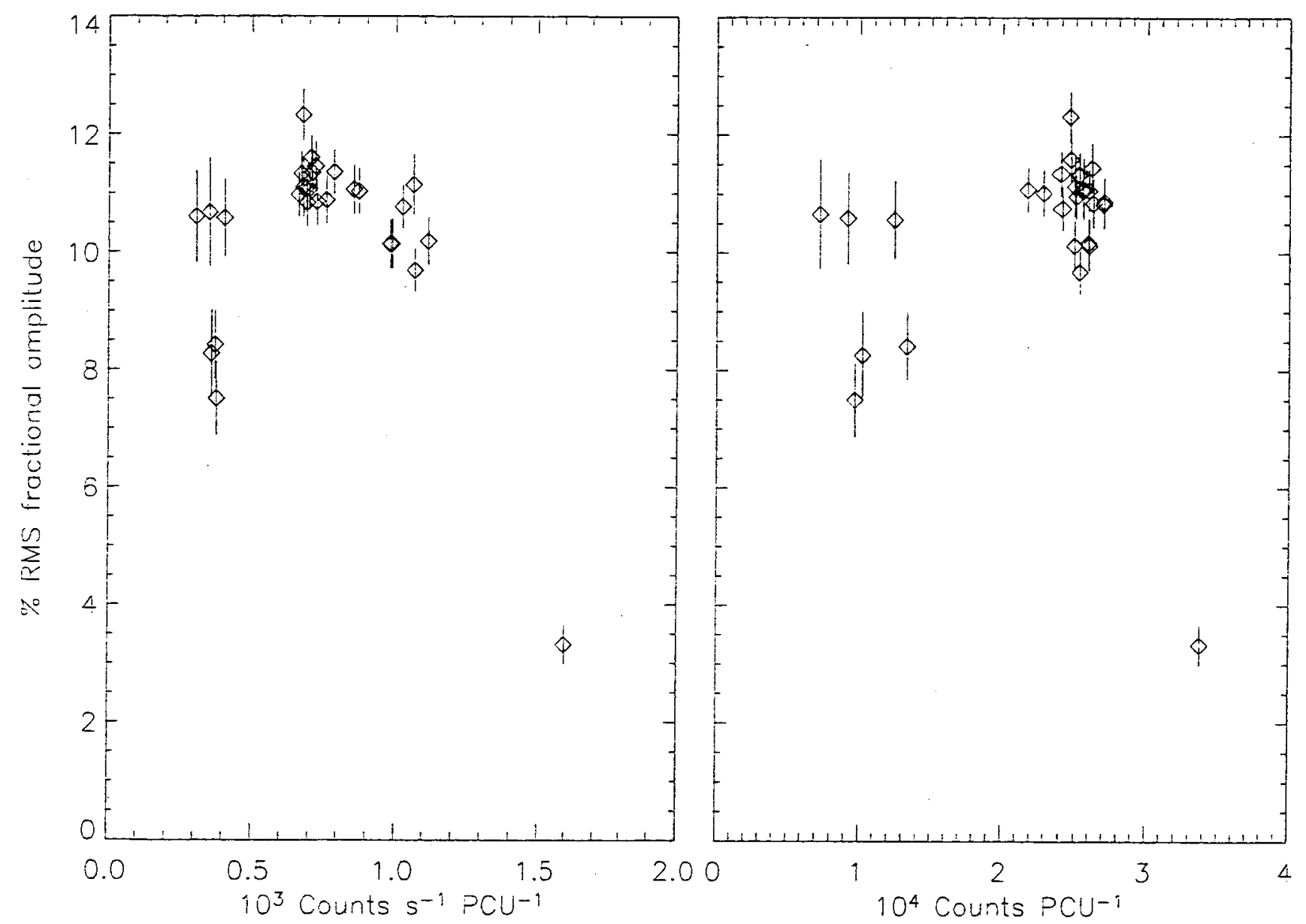

Fig. 12. - The left panel shows accretion-corrected fundamental fractional amplitude of the bursts against peak burst flux (corrected for background and persistent emission). The right panel shows the behavior against total burst fiux (corrected for background and persistent emission). 

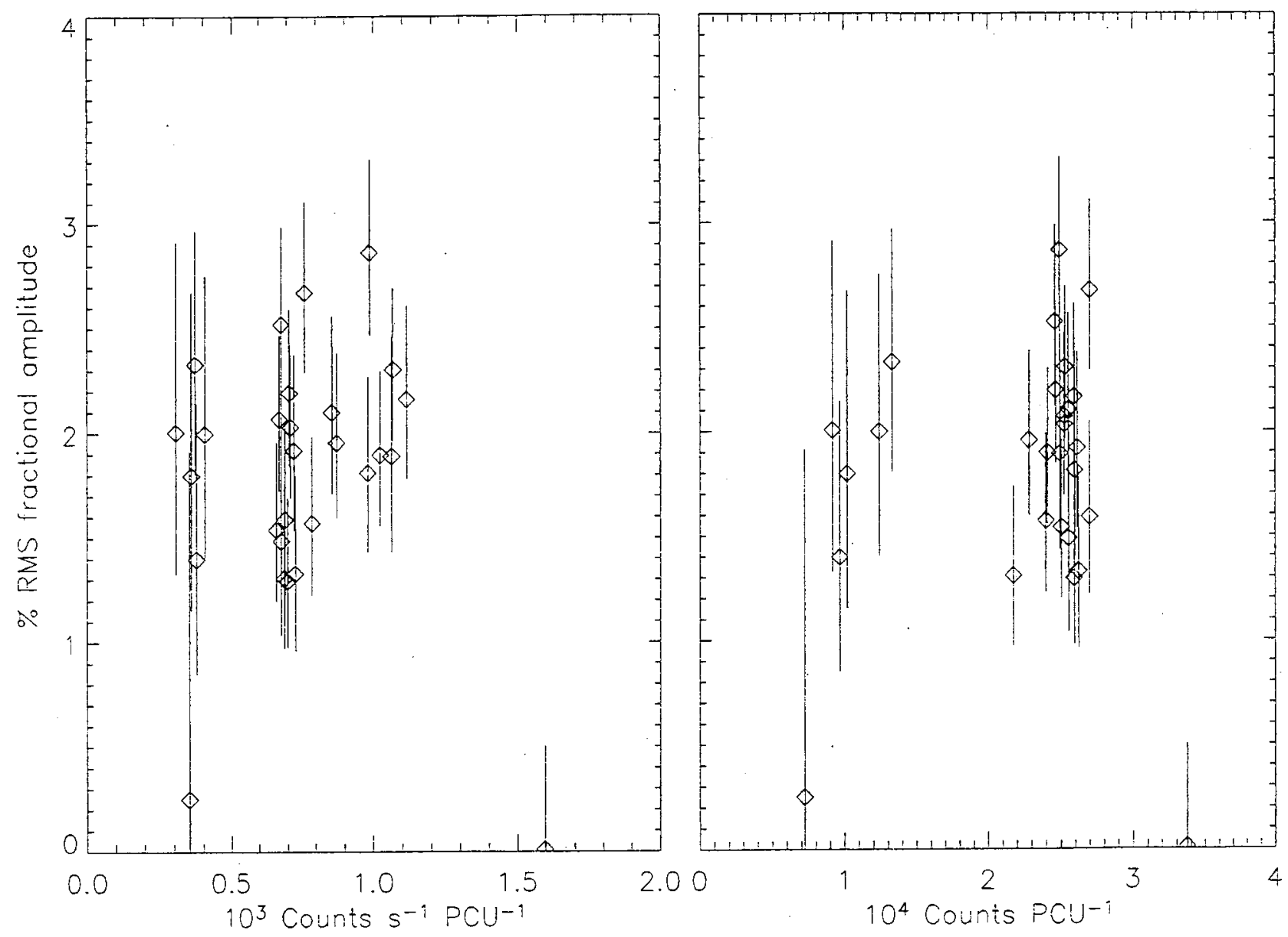

Fig. 13. - The left panel shows accretion-corrected first harmonic fractional amplitude of the bursts against peak burst flux (corrected for background and persistent emission). The right panel shows the behavior against total burst flux (corrected for background and persistent emission). 

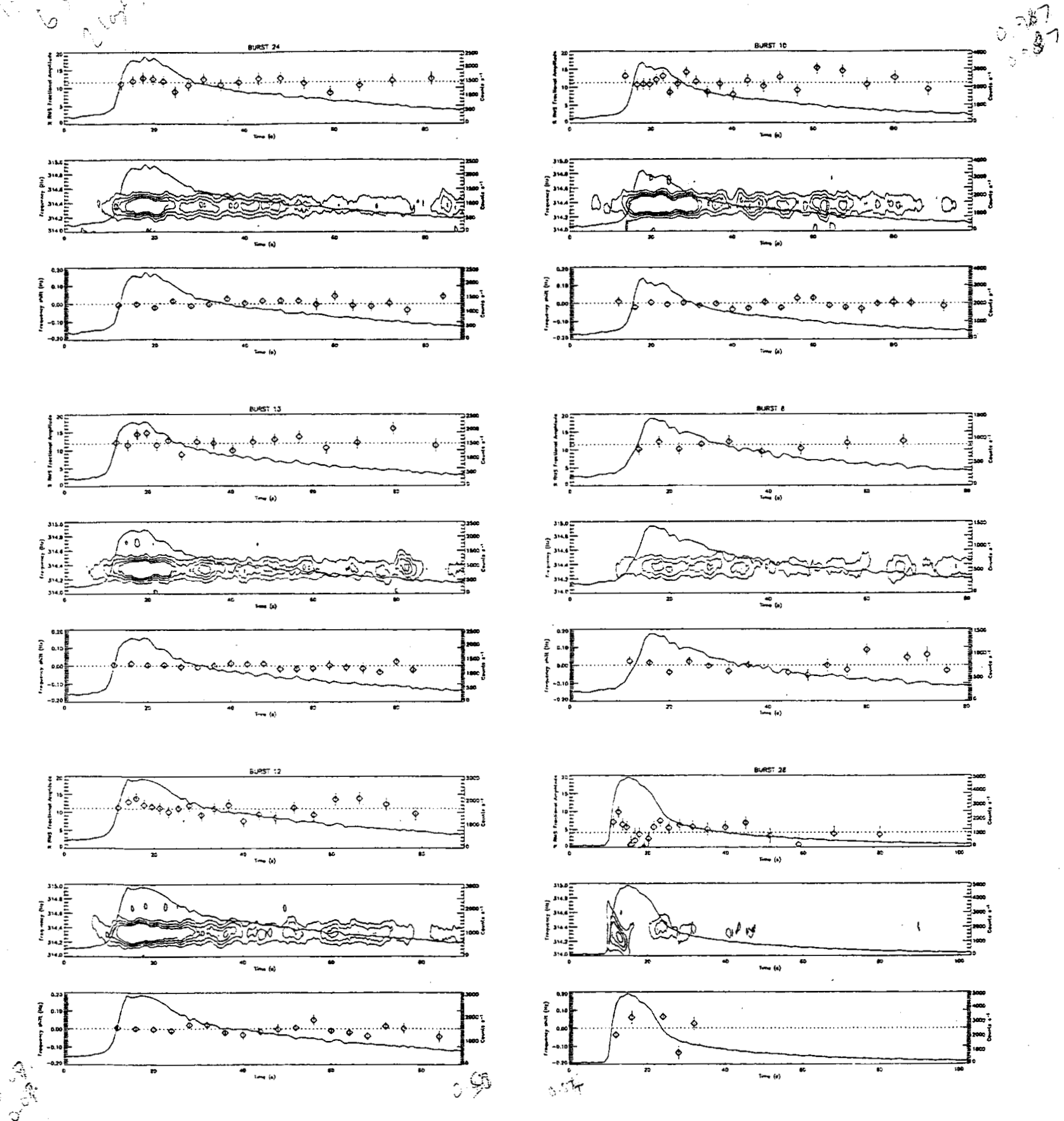

Fig. 14. - Light curves for several of the bursts. The upper panel shows fractional amplitude of the fundamental, calculated for non-overlapping bins of 5000 photons. The dotted line indicates the burst average fractional amplitude. The center panel shows the dynamical power spectrum, calculated using overlapping $4 \mathrm{~s}$ bins, with new bins starting at 0.25 s intervals. The contours show $Z_{1}^{2}=15,35,55,75$. The lower panel shows the difference between the peak frequency (calculated for non-overlapping $4 \mathrm{~s}$ bins and a minimum signal strength $Z_{m}=15$ ) and the best-fit orbital frequency. The error bars shown in the plots are the equivalent of $1 \sigma$ error bars: there is further discussion of error bars elsewhere in the paper. Burst 24 has the lowest amplitude variability; Burst 10 one of the highest. Burst 13 has the lowest frequency variability; Burst 8 one of the highest. Bursts 12 and 28 are included 


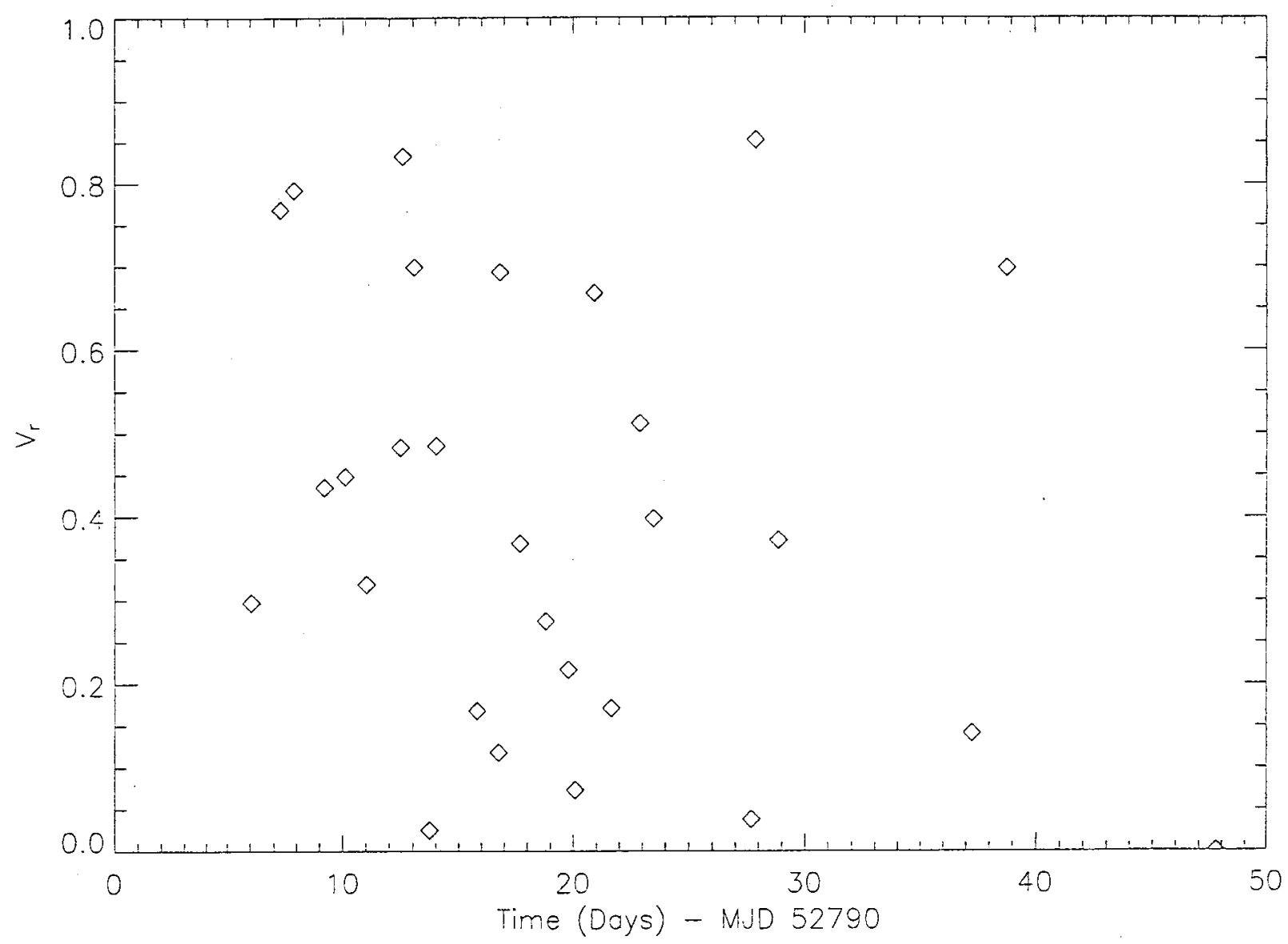

Fig. 15.- Fractional amplitude variability of each burst as quantified by $V_{r}$. Low values indicate highly variable bursts, high values low variability bursts. Note that there are no bursts for which $V_{r}$ exceeds 0.852 . If the null (constant fractional amplitude) hypothesis were true one would expect about 4 bursts in this range. 


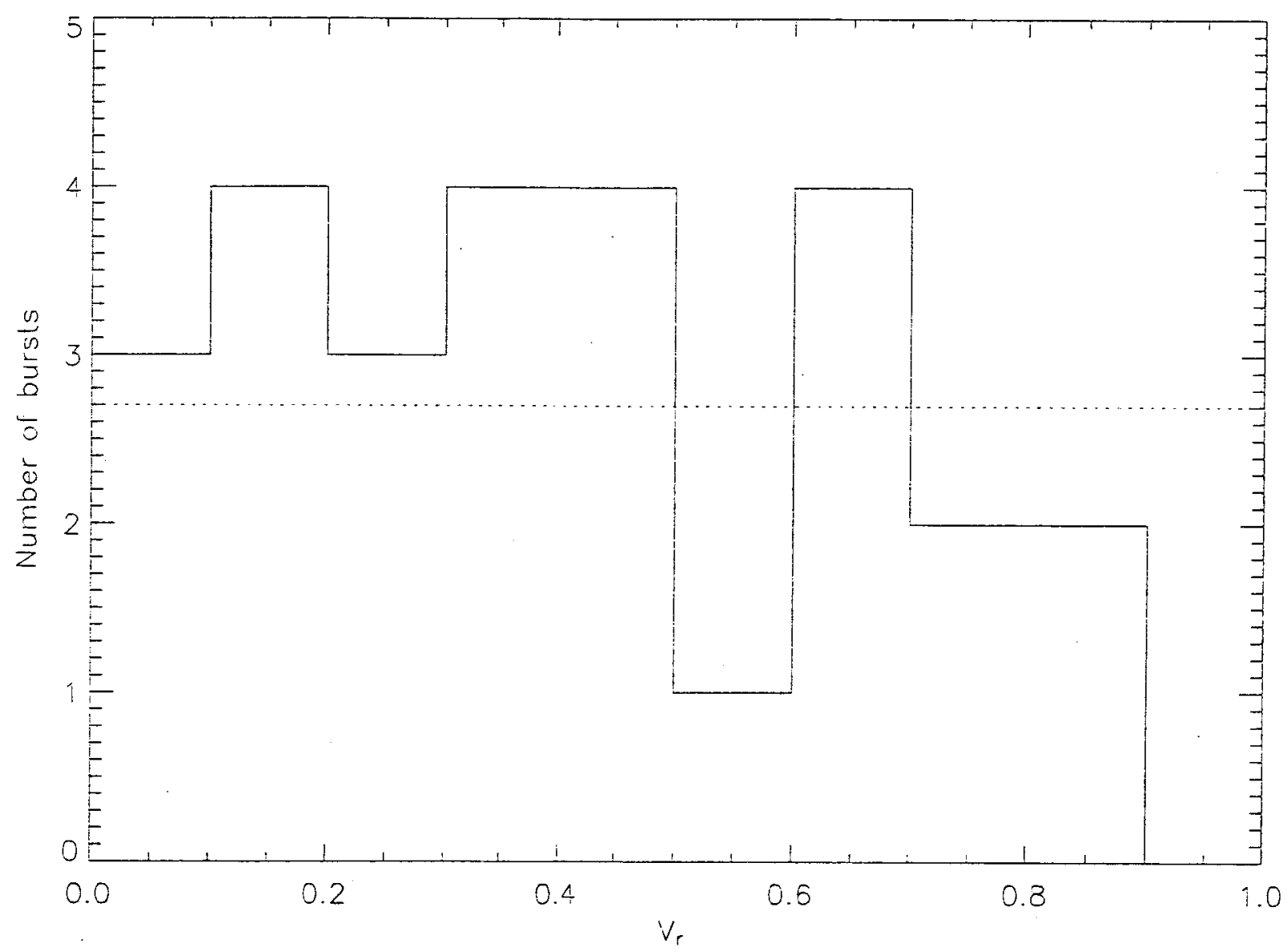

Fig. 16.- Histogram showing the distribution of the $V_{r}$ values for the first 27 bursts. The dashed line indicates the distribution that we would expect if there were no variation in fractional amplitude during the bursts. The histogram shows that there seems to be a shift towards lower values of $V_{r}$, suggesting some variability in fractional amplitude. 


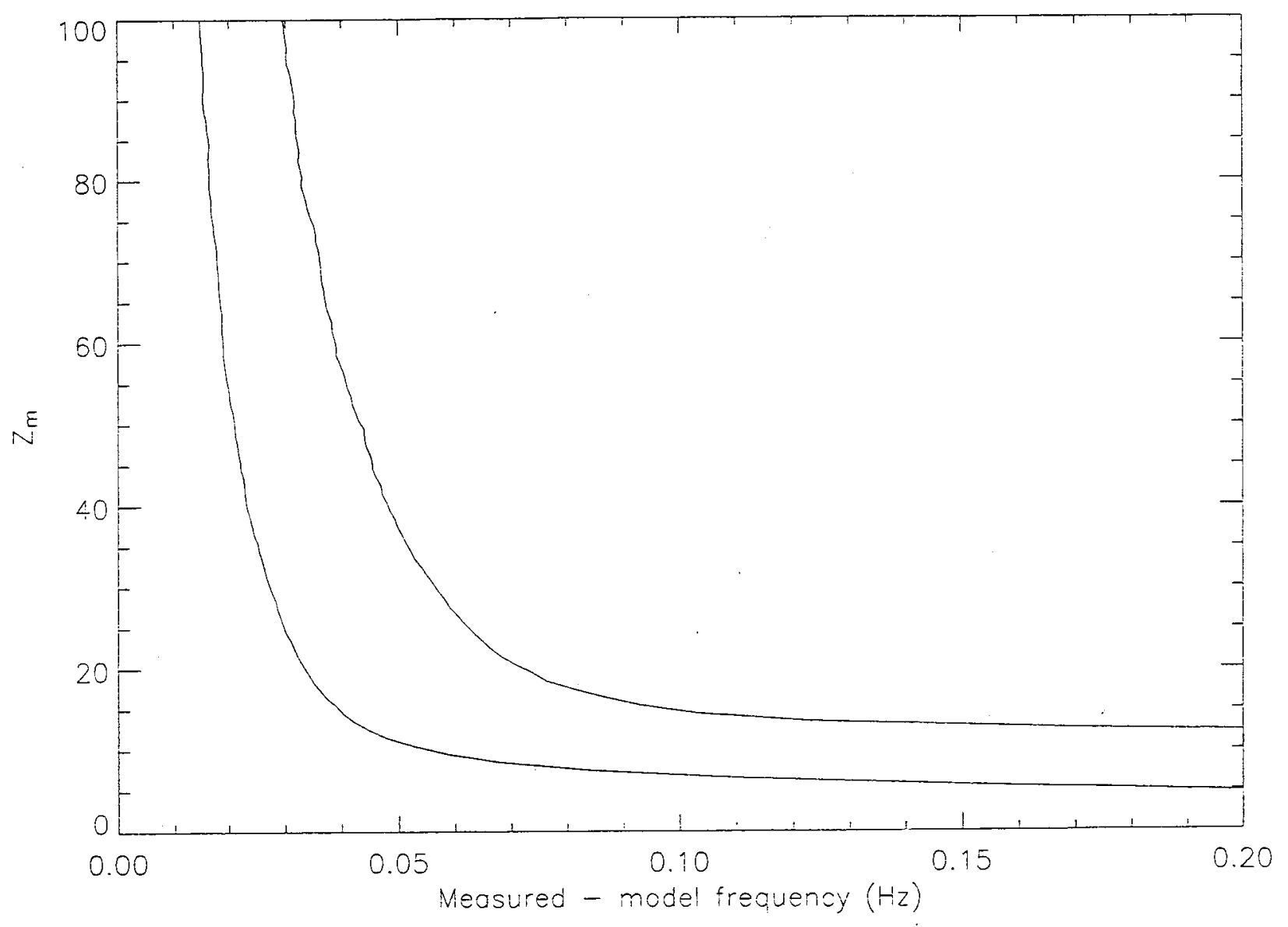

Fig. 17.- Frequency distributions derived from Monte Carlo simulations, for independent time bins of $4 \mathrm{~s}$ duration. The lines shown are $1 \sigma$ and $2 \sigma$ error bars. 


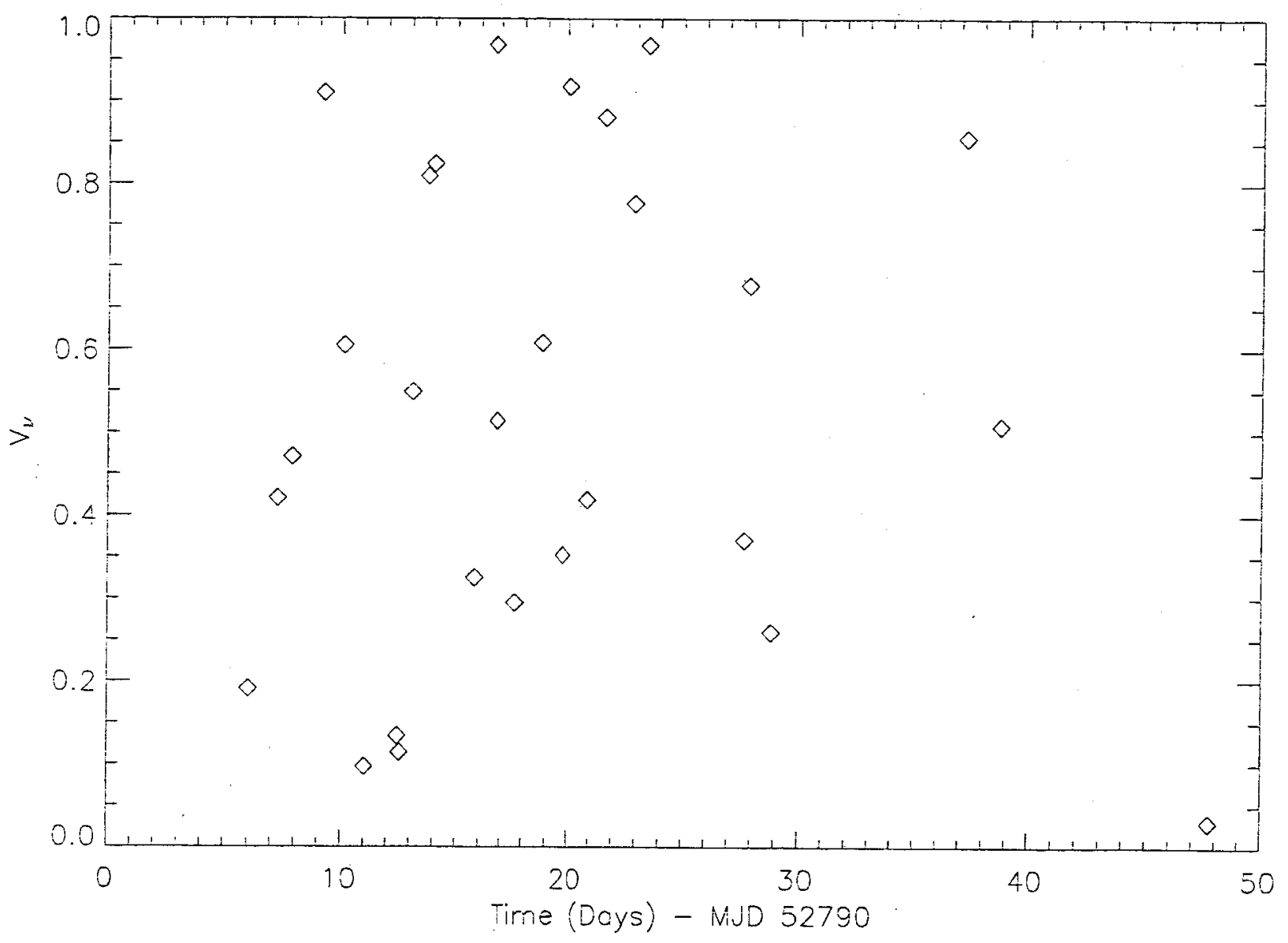

Fig. 18.- Frequency variability of each burst as measured by $V_{\nu}$. Low values indicate highly variable bursts, high values low variability bursts. 


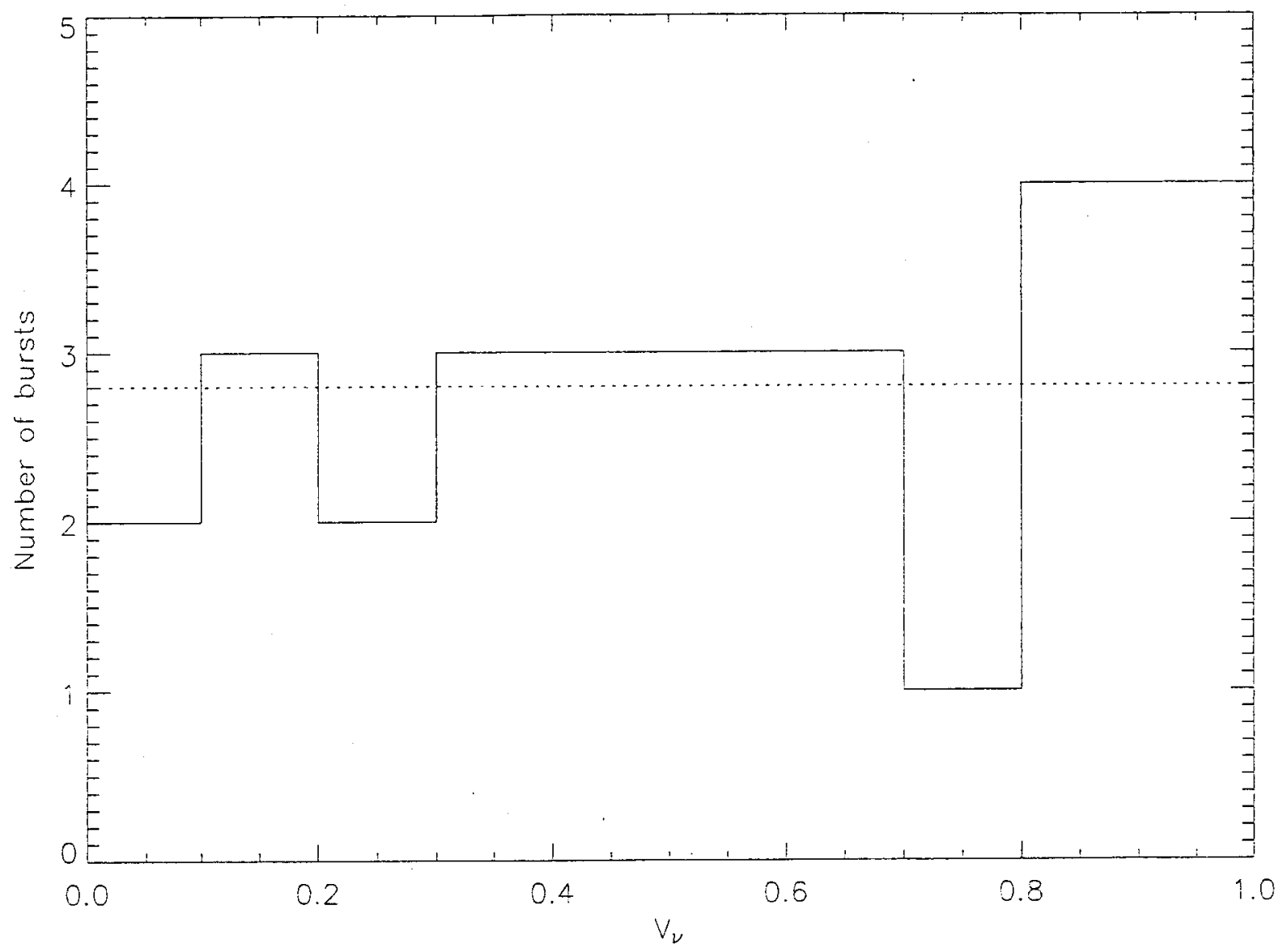

Fig. 19.- Histogram showing the distribution of the $V_{\nu}$ values for all 28 bursts. The dashed line indicates the distribution that we would expect if there were no variation in frequency during the bursts. There is no skew towards lower values of $V_{\nu}$, as we would expect if there was frequency variability. 


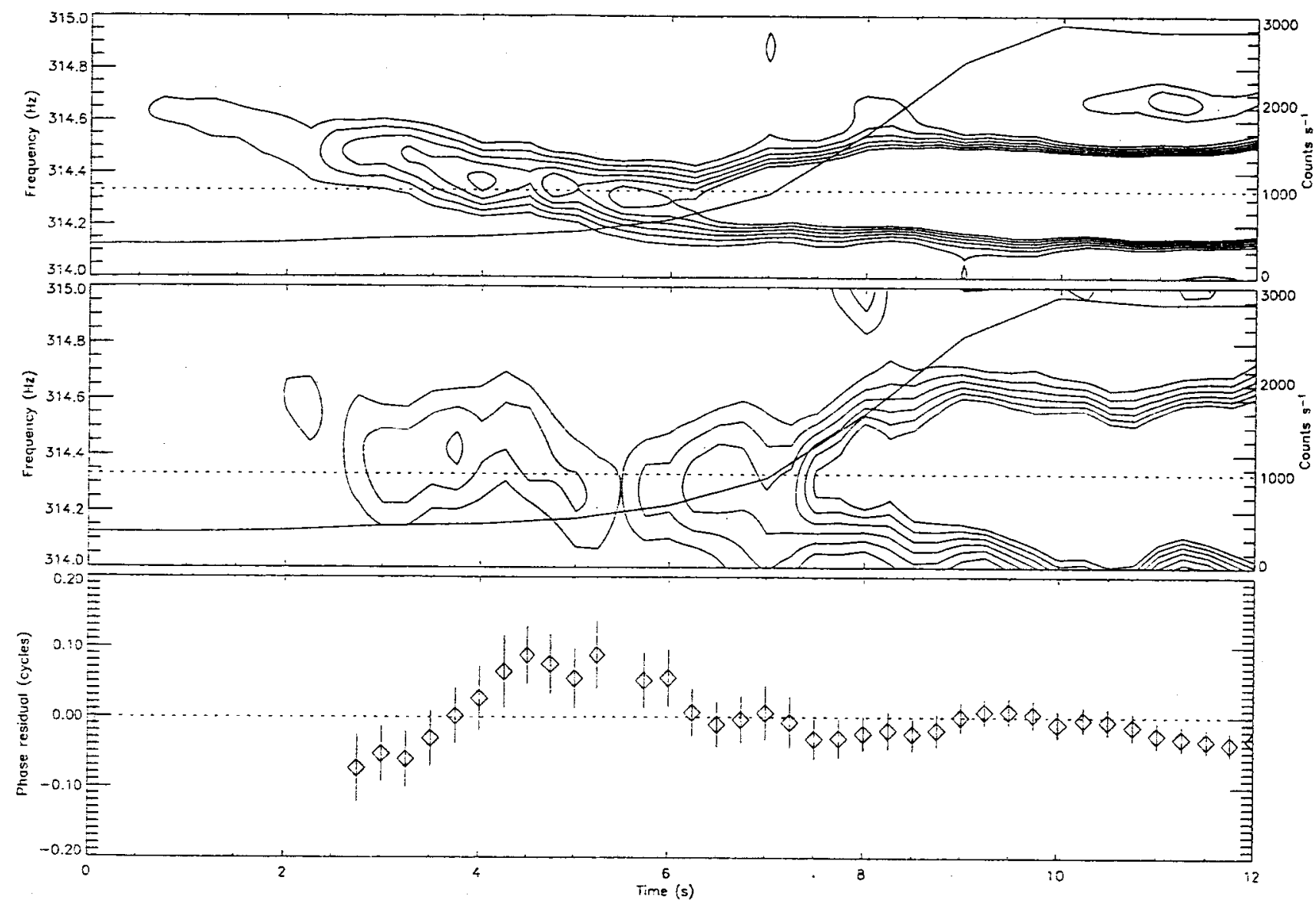

Fig. 20.- The burst rise of Burst 12. The upper panel shows the dynamical power spectrum for $4 \mathrm{~s}$ bins, with a new bin starting every $0.25 \mathrm{~s}$. The center panel shows the dynamical power spectrum for $2 \mathrm{~s}$ bins, with a new bin starting every $0.25 \mathrm{~s}$. The contour levels are $Z_{m}=10,15,20,25,30,35$. The lower panel shows phase residuals compared to the best fit orbital frequency evolution model, for $2 \mathrm{~s}$ bins with new bins starting every $0.25 \mathrm{~s}$. 

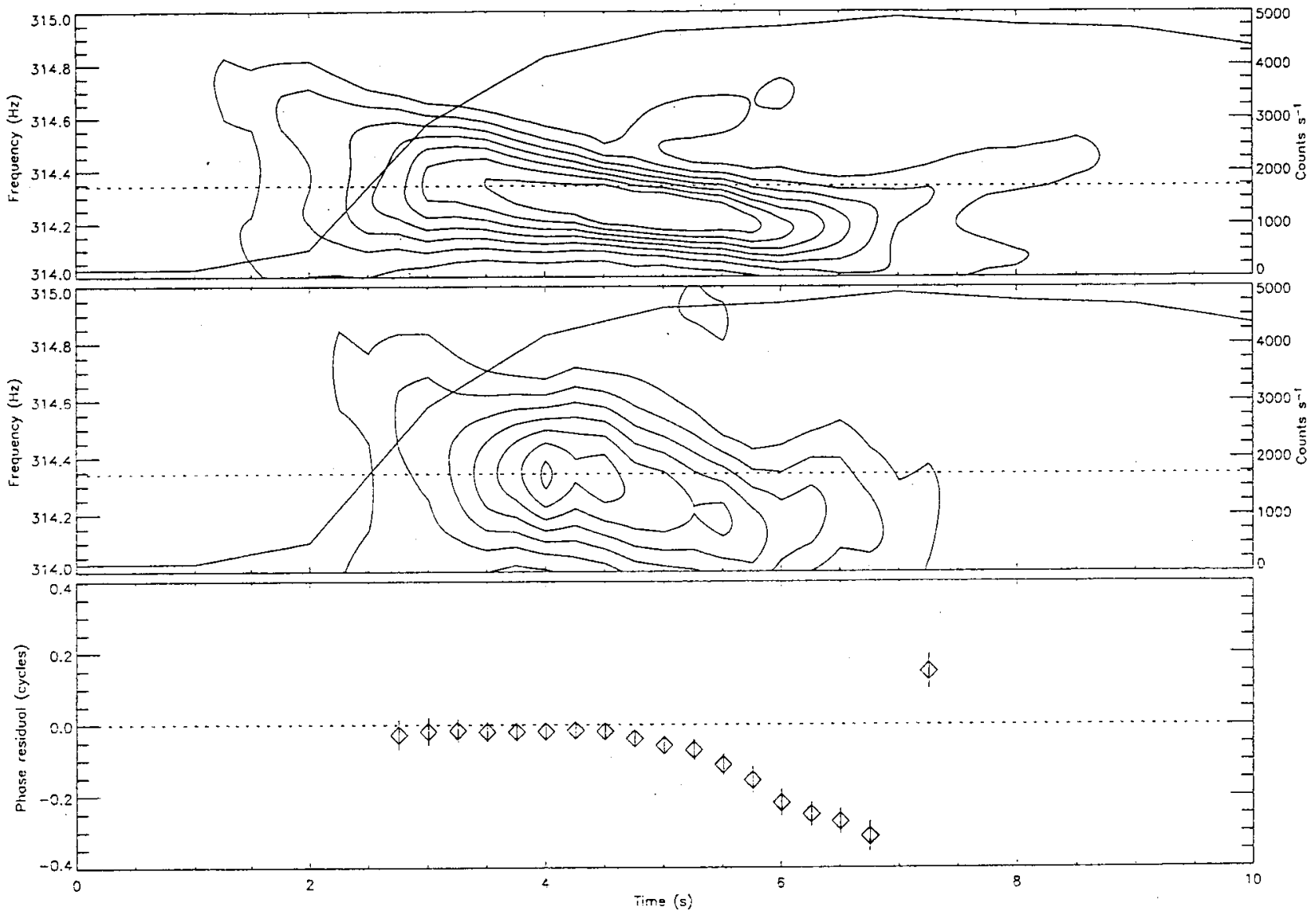

Fig. 21.- The burst rise of Burst 28. The upper panel shows the dynamical power spectrum for $4 \mathrm{~s}$ bins, with a new bin starting every $0.25 \mathrm{~s}$. The center panel shows the dynamical power spectrum for $2 \mathrm{~s}$ bins, with a new bin starting every $0.25 \mathrm{~s}$. The contour levels are $Z_{m}=10,20,30,40,50,60,70$. The lower panel shows phase residuals compared to the best fit orbital frequency evolution model, for $2 \mathrm{~s}$ bins with new bins starting every $0.25 \mathrm{~s}$. 
Table 1. Summary of burst properties

\begin{tabular}{|c|c|c|c|c|c|c|c|}
\hline \multirow[t]{2}{*}{ Index } & \multirow{2}{*}{$\begin{array}{c}\text { Peak Time } \\
\text { (Days - MJD 52790) }\end{array}$} & \multirow{2}{*}{$\begin{array}{c}\text { Peak flux } \\
\left(10^{2} \mathrm{Cts} \mathrm{s}^{-1} \mathrm{PCU}^{-1}\right)\end{array}$} & \multirow{2}{*}{$\begin{array}{c}\nu \\
(\mathrm{Hz})\end{array}$} & \multicolumn{2}{|c|}{ RMS fractional amplitude (\%) } & \multicolumn{2}{|c|}{ Variability } \\
\hline & & & & Fundamental & 1st harmonic & $V_{r}$ & $V_{f}$ \\
\hline 1 & 6.0407 & 3.8 & 314.314 & $7.8 \pm 0.5$ & $1.7_{-0.5}^{+0.6}$ & 0.297 & 0.191 \\
\hline 2 & 7.2687 & 9.9 & 314.307 & $10.1 \pm 0.4$ & $2.9_{-0.4}^{+0.4}$ & 0.768 & 0.421 \\
\hline 3 & 7.8836 & 11.1 & 314.404 & $10.1 \pm 0.4$ & $2.3_{-0.3}^{+0.4}$ & 0.792 & 0.471 \\
\hline 4 & 9.1963 & 10.7 & 314.338 & $9.7=0.3$ & $2.4_{-0.3}^{\div 0.4}$ & 0.435 & 0.910 \\
\hline $\bar{j}$ & 10.0993 & 10.7 & 314.319 & $11.0 \pm 0.5$ & $2.0 \div 0.5$ & 0.448 & 0.605 \\
\hline 6 & 11.0293 & 3.6 & 314.316 & $8.7 \doteq 0.6$ & $2.0_{-0.5}^{+0.7}$ & 0.319 & 0.097 \\
\hline 7 & 12.4664 & 7.1 & 314.335 & $11.4 \doteq 0.3$ & $2.3_{-0.3}^{+0.3}$ & 0.483 & 0.134 \\
\hline 8 & 12.5633 & 4.0 & 314.365 & $10.8 \doteqdot 0.6$ & $2.3_{-0.5}^{\div 0.6}$ & 0.833 & 0.114 \\
\hline 9 & 13.0594 & 3.7 & 314.405 & $8.8 \pm 0.5$ & $2.4 \div 0.5$ & 0.699 & 0.549 \\
\hline 10 & 13.7342 & 7.9 & 314.370 & $11.4 \doteq 0.3$ & $1.7_{-0.3}^{\div 0.4}$ & 0.025 & 0.809 \\
\hline 11 & 14.0145 & 8.7 & 314.324 & $11.0 \doteq 0.3$ & $2.0_{-0.3}^{\div 0.4}$ & 0.485 & 0.824 \\
\hline 12 & 15.7895 & 6.6 & 314.331 & $11.0 \doteq 0.3$ & $1.6_{-0.3}^{\div 0.4}$ & 0.168 & 0.325 \\
\hline 13 & 16.7475 & 6.8 & 314.346 & $12.2 \doteq 0.4$ & $2.5_{-0.3}^{+0.4}$ & 0.118 & 0.968 \\
\hline 14 & 16.8181 & 3.5 & 314.396 & $10.7 \pm 0.8$ & $0.8_{-0.8}^{+1.4}$ & 0.693 & 0.514 \\
\hline 15 & 17.6670 & 7.1 & 314.393 & $11.2 \doteq 0.3$ & $2.1_{-0.3}^{\div 0.3}$ & 0.368 & 0.295 \\
\hline 16 & 18.7955 & 7.3 & 314.368 & $10.8 \pm 0.4$ & $1.5 \div 0.4$ & 0.275 & 0.508 \\
\hline 17 & 19.7817 & 7.6 & 314.356 & $10.8=0.4$ & $2.7_{-0.3}^{\div 0.4}$ & 0.217 & 0.352 \\
\hline 18 & 20.0682 & 10.2 & 314.327 & $10.6=0.3$ & $2.0 \div 0.4$ & 0.073 & 0.918 \\
\hline 19 & 20.9015 & 3.1 & 314.407 & $10.6 \pm 0.6$ & $2.1_{-0.5}^{\div 0.7}$ & 0.668 & 0.419 \\
\hline 20 & 21.6396 & 9.8 & 314.378 & $10.2 \pm 0.4$ & $1.9_{-0.3}^{\div 0.4}$ & 0.171 & 0.881 \\
\hline 21 & 22.8887 & 8.5 & 314.375 & $11.0 \pm 0.4$ & $2.2 \div 0.4$ & 0.512 & 0.777 \\
\hline 22 & 23.4688 & 6.7 & 314.308 & $11.3 \pm 0.3$ & $2.1_{-0.3}^{+0.3}$ & 0.398 & 0.968 \\
\hline 23 & 27.6937 & 6.9 & 314.379 & $11.1 \pm 0.3$ & $1.4_{-0.3}^{+0.4}$ & 0.038 & 0.370 \\
\hline 24 & 27.8834 & 7.2 & 314.360 & $11.4 \pm 0.4$ & $2.0_{-0.3}^{\div 0.4}$ & 0.852 & 0.678 \\
\hline 25 & 28.8537 & 6.8 & 314.338 & $11.1 \pm 0.5$ & $1.6_{-0.4}^{+0.5}$ & 0.372 & 0.259 \\
\hline 26 & 37.2535 & 7.0 & 314.383 & $11.1 \pm 0.3$ & $1.4_{-0.3}^{+0.3}$ & 0.141 & 0.856 \\
\hline 27 & 38.7935 & 6.9 & 314.305 & $10.9 \pm 0.4$ & $1.6_{-0.3}^{+0.4}$ & 0.698 & 0.508 \\
\hline 28 & 47.7381 & 16.0 & 314.353 & $3.5 \pm 0.3$ & $0.0^{+0.5}$ & $<10^{-4}$ & 0.030 \\
\hline
\end{tabular}

